Please do not remove this page

RMIT

UNIVERSITY

\title{
Fluttering energy harvesters in the wind: A review
}

McCarthy, Jesse; Watkins, Simon; Deivasigamani, Arvind; John, Sabu

https://researchrepository.rmit.edu.au/esploro/outputs/9921862856601341/filesAndLinks?institution=61RMIT_INST\&index=null

McCarthy, J., Watkins, S., Deivasigamani, A., \& John, S. (2016). Fluttering energy harvesters in the wind: A review. Journal of Sound and Vibration, 361, 355-377. https://doi.org/10.1016/j.jsv.2015.09.043

Document Version: Accepted Manuscript

Published Version: https://doi.org/10.1016/j.jsv.2015.09.043

Repository homepage: https://researchrepository.rmit.edu.au (c) 2015 Elsevier Ltd. All rights reserved.

Downloaded On 2023/04/27 01:34:18 +1000

Please do not remove this page 
Thank you for downloading this document from the RMIT Research Repository.

The RMIT Research Repository is an open access database showcasing the research outputs of RMIT University researchers.

RMIT Research Repository: http://researchbank.rmit.edu.au/

\section{Citation:}

McCarthy, J, Watkins, S, Deivasigamani, A and John, S 2016, 'Fluttering energy harvesters in the wind: A review', Journal of Sound and Vibration, vol. 361, pp. 355-377.

See this record in the RMIT Research Repository at:

https://researchbank.rmit.edu.au/view/rmit:33768

Version: Accepted Manuscript

Copyright Statement: (c) 2015 Elsevier Ltd. All rights reserved. Creative Commons Attribution-NonCommercial-NoDerivatives 4.0 International License.

Link to Published Version:

http://dx.doi.org/10.1016/j.jsv.2015.09.043 


\title{
Fluttering Energy Harvesters in the Wind: A Review
}

\author{
J. M. McCarthy ${ }^{\mathrm{a}, *}$, S. Watkins ${ }^{\mathrm{a}}$, A. Deivasigamani ${ }^{\mathrm{a}}$, S. J. John ${ }^{\mathrm{a}}$ \\ ${ }^{a}$ RMIT University, School of Aerospace, Mechanical E Manufacturing Engineering, PO Box 71, Bundoora, Victoria 3083, Australia
}

\begin{abstract}
The growing area of harvesting energy by aerodynamically-induced flutter in a fluid stream is reviewed. Numerous approaches were found to understand, demonstrate and [sometimes] optimise harvester performance based on Movement-Induced or Extraneously-Induced Excitation. Almost all research was conducted in smooth, unidirectional flow domains; either experimental or computational. The power outputs were found to be very low when compared to conventional wind turbines, but potential advantages could be lower noise levels. A consideration of the likely outdoor environment for fluttering harvesters revealed the flow would be highly turbulent and having a mean flow angle in the horizontal plane that could approach a harvester from any direction. Whilst some multiple harvester systems in smooth, well-aligned flow found enhanced efficiency (due to beneficial wake interaction) this would require an invariant flow approach angle. It was concluded that further work needs to be performed to find a universally accepted metric for efficiency and to understand the effects of the realities of the outdoors, including the highly variable and turbulent flow conditions likely to be experienced.
\end{abstract}

Keywords: Flutter energy harvesting, turbulence, piezoelectric, wind velocity

\section{Introduction and background}

Energy extracted from the wind has been, and would appear to continue to be a vital source of renewable energy. Traditionally, wind-energy transduction to mechanical or electrical energy has been accomplished through the use of wind turbines. Multiple kilo- or mega-watt class turbines are often arranged together in outdoor areas and strategically placed to maximise harvesting potential. Energy supply to power grids from these wind farms is increasing steadily around the world. As of 2010, worldwide wind-energy capacity reached $196 \mathrm{MW}$ and is projected to reach 1,500 MW by the year 2020 [1]. More recently, wind turbines have been scaled down in size for deployment into urban or suburban environments. Despite continued developments, there have been concerns with urban-based wind turbines on the basis of noise, vibration, cost-effectiveness and safety [e.g. 2-4], which have led researchers to investigate novel methods of extracting wind energy in urban environments, including the exploitation of aeroelastic flutter.

Aeroelastic flutter constitutes only one of many classes of aeroelastic mechanisms that may occur in dynamic Fluid-Structure Interactions (FSIs), as illustrated in Fig. 1 through the concept of the "triangle of forces" proposed by [5]. However, given the context of this review, we restrict ourselves to applications involving flutter, where the aerodynamic and elastic forces dominate. Flutter has traditionally been classified as a destructive phenomena by the aeroelasticity research community, and is a highly non-linear problem due to the large strains and geometric deformations present, as well as the transient behaviour. One of the first documented cases of flutter traces back to the Handley Page 0/400 bomber in World War I, where self-excited fuselage- and tail-flutter would occur due to modal coalescence between two low-frequency modes [6]. A more well-known example of flutter-induced destruction is the Tacoma Narrows bridge collapse in 1940, where torsional flutter at sufficiently large amplitudes caused catastrophic failure of the entire bridge.

\footnotetext{
${ }^{*}$ To whom correspondence should be addressed.

Email address: jesse.mccarthy@rmit.edu.au (J. M. McCarthy)
} 


\begin{tabular}{|c|c|c|c|}
\hline \multicolumn{4}{|c|}{ Nomenclature } \\
\hline \multicolumn{2}{|l|}{ Variables } & St & Strouhal number \\
\hline$A_{E A}$ & Harvester electro-active area & $\overline{U_{z}}$ & Mean flow speed at height $z$ \\
\hline$A_{\text {surface }}$ & Harvester surface area & $U$ & Flow speed \\
\hline$A_{\text {swept }}$ & Harvester swept area & $u, v, w$ & Streamwise, cross-stream and vertical velocities \\
\hline$b$ & Beam width & $u^{\prime}, v^{\prime}, w^{\prime}$ & Streamwise, cross-stream and vertical fluctuating veloc- \\
\hline C & Capacitance & & ities \\
\hline$D$ & Characteristic bluff-body dimension & $U_{c}$ & Critical flutter speed \\
\hline$f_{s}$ & Vortex shedding frequency & $V$ & Harvester volume \\
\hline$h$ & Beam thickness & $V_{R M S}$ & Root-mean-square voltage \\
\hline$I_{u, v, w}$ & Turbulence intensity of $u, v$ or $w$ velocities & $x, y, z$ & Streamwise, cross-stream and vertical distances \\
\hline$k$ & Material dependent constant & Y & Elastic modulus \\
\hline$L$ & Beam length & \multicolumn{2}{|c|}{ Greek Symbols } \\
\hline$m_{p}$ & Beam mass per unit length & $\eta_{1}$ & Hydrodynamic efficiency \\
\hline$P$ & Power & $\eta_{2}$ & Electromechanical efficiency \\
\hline$P_{A}$ & Power available in fluid flow & $\eta_{3}$ & Electrical subsystem efficiency \\
\hline$P_{B}$ & Power delivered to beam by flow & $\eta_{C}$ & Capture efficiency \\
\hline$P_{\text {Area }}$ & Harvester area-based power density & $\eta_{\text {Betz }}$ & Betz limit, equal to 0.593 \\
\hline$P_{E A A}$ & Harvester electro-active area-based power density & $\eta_{i s o}$ & Efficiency isoline \\
\hline$P_{\text {mean }}$ max & Maximum mean power & $\mu$ & Mass ratio \\
\hline$P_{\text {mean }}$ & Mean power & $v$ & Kinematic viscosity \\
\hline$P_{\text {Volume }}$ & Harvester volume-based power density & $\rho_{f}$ & Fluid density \\
\hline$\tilde{q}_{\max }$ & Experimental beam maximum amplitude & $\rho_{s}$ & Beam density \\
\hline & Theoretical beam maximum amplitude & $\phi^{t i p}$ & $\begin{array}{l}\text { Maximum tip displacement of the normalised mode } \\
\text { shape }\end{array}$ \\
\hline$\overline{R_{v}}$ & $\sqrt{\sqrt{u^{2}+v^{2}+w^{2}}}$ & $\phi_{2}$ & Normalised mode shape \\
\hline$R_{L}$ & Load resistance & $\omega$ & Flutter frequency \\
\hline$R_{L_{o p t}}$ & Optimum load resistance & \multicolumn{2}{|c|}{ Subscripts } \\
\hline $\operatorname{Re}$ & Reynolds number & $R M S$ & Root Mean Square \\
\hline
\end{tabular}

Piezoelectric materials are a class of materials that, given mechanical strain, output an electric charge, or vice versa ${ }^{1}$. These materials have generally been deployed in dynamic environments to sense, actuate, or to scavenge energy. Thus, during flutter, a compliant piezoelectric material could transduce fluidic kinetic energy to electrical energy. Another approach is to exploit flutter motions in an electromagnetic system to induce a changing magnetic field, generating electricity. One of the earliest known studies on piezoelectric flutter harvesters was by [7], where a thin beam bonded with polyvinylidene-fluoride (PVDF) piezoelectric patches was placed in the near wake of a bluff body. Particle Image Velocimetry (PIV) was conducted and the data suggested that the beam flutter frequency could 'lock in' with the bluff-body vortex shedding. Since this study, it has become clear that effective design and deployment of a piezoelectric harvester requires a detailed understanding of the mechanism of flutter, and the electromechanical dynamics inherent in such a system.

In this review, we aim to provide an overview of the work done in the area of piezoelectric energy harvesting utilising flutter. In section 2, we explore two different types of flutter and their relevance to energy harvesting and in section 3, we review the realisation of these two methods. In section 4, we examine the wind characteristics relevant to flutter harvesting within the earth's Atmospheric Boundary Layer (ABL). In section 5, we discuss work that has been done to address other components of flutter harvesters, as well as review different harvester performance metrics.

\footnotetext{
${ }^{1}$ The former is the direct piezoelectric effect, the latter is the converse piezoelectric effect; i.e. where an electric charge is applied to the piezoelectric material and strain results.
} 


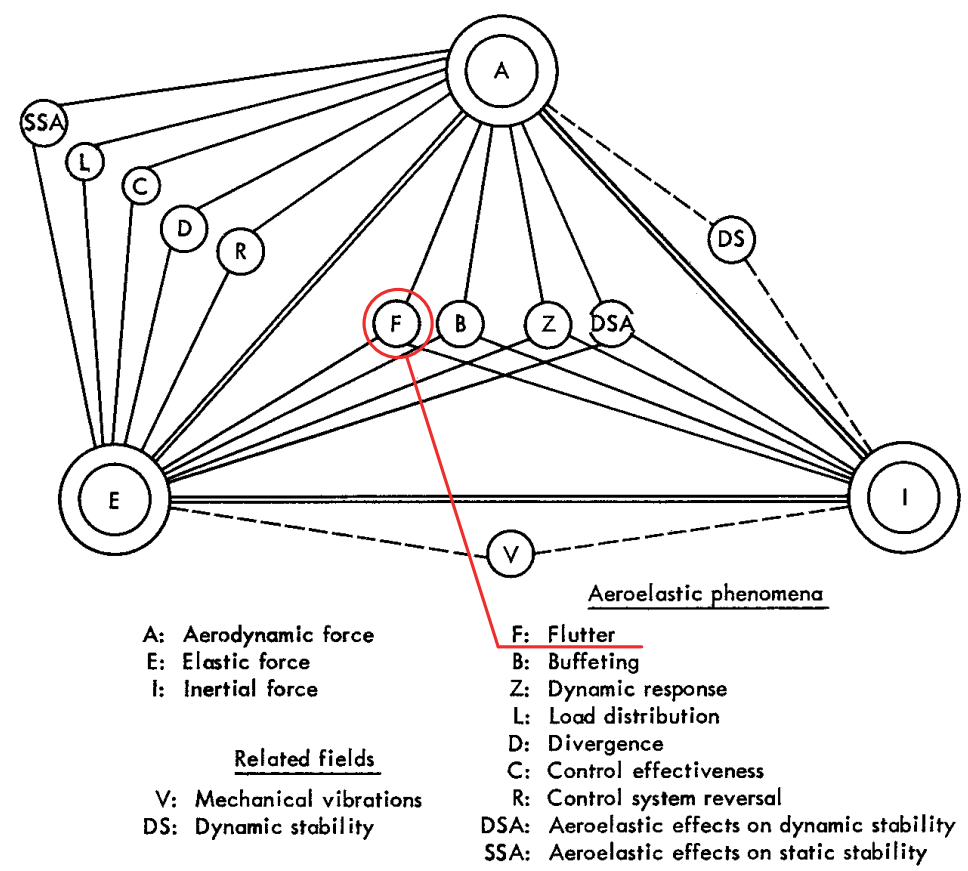

Figure 1: The aeroelastic triangle of forces with flutter highlighted, in the context of aircraft analysis. Taken from [5].

Finally, in section 6 we present a discussion and concluding remarks.

\subsection{A note on performance metrics}

Flutter energy harvesting is a relatively novel field, and one still without a standardised technical method by which to represent harvester performance and efficiency. Thus, we think it expedient to introduce some mathematical terms used across the current literature for defining the performance of a flutter harvester, prior to the review.

The output of interest from a harvester is the mean power, $P_{\text {mean }}$. In order to normalise the measured power, there have generally been three different definitions of power density, shown in Eqs. (1), (2) and (3);

$$
\begin{aligned}
& P_{\text {Area }}=\frac{P_{\text {mean }}}{A_{\text {surface }}}, \\
& P_{\text {EAA }}=\frac{P_{\text {mean }}}{A_{E A}}, \\
& P_{\text {Volume }}=\frac{P_{\text {mean }}}{V},
\end{aligned}
$$

where $P_{\text {Area }}$ is the power density based on the total surface area of the harvester $A_{\text {surface }}, P_{E A A}$ is the power density based on the electro-active area of the harvester ${ }^{2} A_{E A}$, and $P_{V o l u m e}$ is the power density based on the total volume of the harvester $V$. Throughout this article, results will be presented as reported from each study, with a detailed discussion of performance metrics conducted in section 5.2.

${ }^{2}$ For example, the piezoelectric area only of a piezoelectric flutter harvester. 


\section{Overview of relevant flutter types}

Flutter is an aeroelastic instability of a compliant structure immersed in a fluid flow. Different flutter types were classified based on the excitation and sustenance mechanisms in $[8,9]$. Flutter of a structure may be instigated by a number of different flow phenomena; however, we restrict our review to two different types of flutter that have been exploited for piezoelectric energy harvesting previously.

\subsection{Movement-Induced Excitation}

Movement-Induced Excitation (MIE) is flutter that is self-excited through a resonant bending instability of the immersed structure $[8,9]$. At a certain flow velocity, usually known as the critical flutter speed, negative damping of the structure occurs and divergence of structural deformations results.

Seminal work by [10] using potential flow methods proved that MIE flutter is a self-excited and self-sustaining phenomenon, with [11] and [12] showing the strong dependence of MIE flutter on length and stiffness of the immersed structure. The work in [10] was extended in [13] and scaling laws were developed for two-dimensional compliant beams in a viscous flow, which showed the relationship between flutter frequency, critical flutter speed and other system parameters, as Eqs. (4) and (5);

$$
\begin{gathered}
\omega \sim \sqrt{\frac{\rho_{f} U^{2}}{\rho_{s} h L}}, \\
U_{c} \sim \sqrt{\frac{Y h^{3}}{\rho_{f} L^{3}}} .
\end{gathered}
$$

Here, $\omega$ is the flutter frequency, $U_{c}$ is the critical flutter speed, $\rho_{f}, \rho_{s}$ the fluid and beam densities respectively, $U$ is the flow speed, $h$ the beam thickness, $L$ the beam length, and $Y$ the beam elastic modulus. More recently, the validity of these scaling laws for three-dimensional, rectangular beams in a wind flow were experimentally verified in [14].

It has been demonstrated that MIE flutter is a combination of in vacuo mode shapes, usually the first and second modes of vibration $[15,16]$. This is true particularly if the non-dimensional mass ratio (Eq. 6) is relatively large;

$$
\mu=\frac{\rho_{s} h}{\rho_{f} L},
$$

where $\mu$ is the mass ratio. It was found in [17] that for sufficiently small mass ratios, the beam flutter motions were governed chiefly by fluid added mass and viscous effects, rather than adhering to predicted modal displacements.

Three distinct regimes of MIE flutter were identified in an analytical and computational study by [18], depending on beam length, stiffness and flow speed:

1. Fixed-point stability, where subsequent to an initial disturbance the system experiences positive damping and the beam returns to a stretched-straight position;

2. Limit-Cycle Oscillations (LCOs), where MIE flutter eventuates and the beam tip displacements closely resemble a sinusoidal function; and

3. Chaotic flapping, where the flutter is characterised by random snap-through events, large increases in drag and a broadband frequency response.

These three regimes may clearly be identified in Fig. 2, which shows the transient response of three beams with differing mass ratios.

In a subsequent study to [18], it was found by [19] that maximum bending strain energy was contained in the LCO regime, while energy outputs decreased significantly when the beam transitioned to chaotic flutter. This constitutes an important finding, since piezoelectric harvesters rely heavily upon the strain generated in the material. 


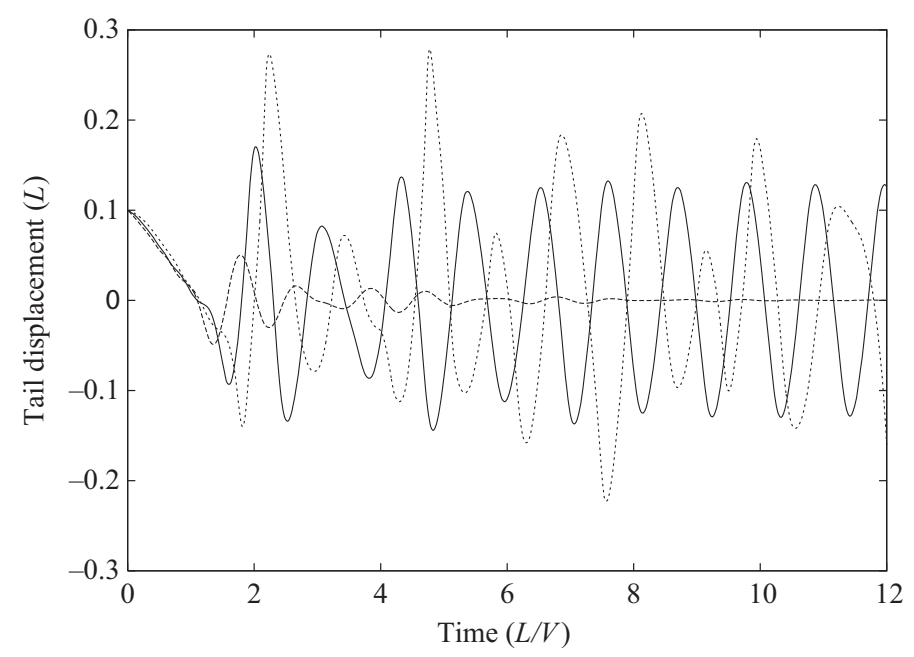

Figure 2: The normalised trailing edge displacement plotted against the non-dimensional time for fixed point stability regime with $\mu=0.025(-$ -), LCO regime with $\mu=0.1$ (-), and chaotic flapping regime with $\mu=0.2(---)$. Taken from [18].

\subsection{Extraneously-Induced Excitation}

A second type of flutter that was classified in [8,9] is Extraneously-Induced Excitation (EIE). EIE flutter of an immersed structure is excited and sustained through external, time-varying flow pressure gradients such as those generated by a von Kárman vortex street. Common types of EIE flutter are Vortex-Induced Vibrations (VIVs), or buffeting of an aircraft near the stall speed (see Fig. 1).

Generally, in the context of energy harvesting, EIE flutter has seen greater utilisation than MIE flutter, despite the need for a more complex setup. Maximising the potential of EIE flutter to harvest wind energy requires matching the dynamics of the flutter harvester to the [upstream] bluff-body vortex shedding. There have been many studies on the vortex shedding of bluff bodies, and comprehensive reviews have been conducted elsewhere [e.g. 20, 21]. Instead, we focus on a few seminal investigations. Fundamental studies in the area of bluff-body vortex shedding were conducted by $[22,23]$, where it was shown experimentally that the Strouhal number (Eq. 7) is some function of the flow Reynolds number (Eq. 8);

$$
\begin{aligned}
& \mathrm{St}=\frac{f_{s} D}{U}, \\
& \mathrm{Re}=\frac{U D}{v},
\end{aligned}
$$

where $\mathrm{St}$ is the non-dimensional Strouhal number, $f_{s}$ is the vortex shedding frequency, $\operatorname{Re}$ is the non-dimensional Reynolds number, $D$ is a characteristic dimension of the bluff body (usually a diameter), and $v$ is the kinematic viscosity of the flow. For subcritical Reynolds numbers, i.e. $300<\operatorname{Re}<3 \times 10^{5}$, the Strouhal number is approximately 0.2 (Fig. 3). Thus, the shedding frequency of the bluff body may be tuned to a resonant frequency of a downstream beam in the near-wake, causing a significant increase in beam deformation amplitudes and subsequent strains and strain rates.

Most work in the area of vortex-shedding analysis has focused on the cases where the prevailing flow direction is orthogonal to the bluff-body streamwise axis, e.g. a circular cylinder placed orthogonally to the streamwise flow direction. Typically, vortex shedding along the streamwise axis of a bluff body is relatively well correlated when the flow is orthogonal $[24,25]$. However, when the bluff body is yawed into or away from the oncoming flow direction, the predicted Strouhal number deviates from the measured Strouhal number because of three dimensional flow effects in the body near wake [26]. In [24], it was observed that for a yaw angle of $60^{\circ}$, the deviation between predicted 


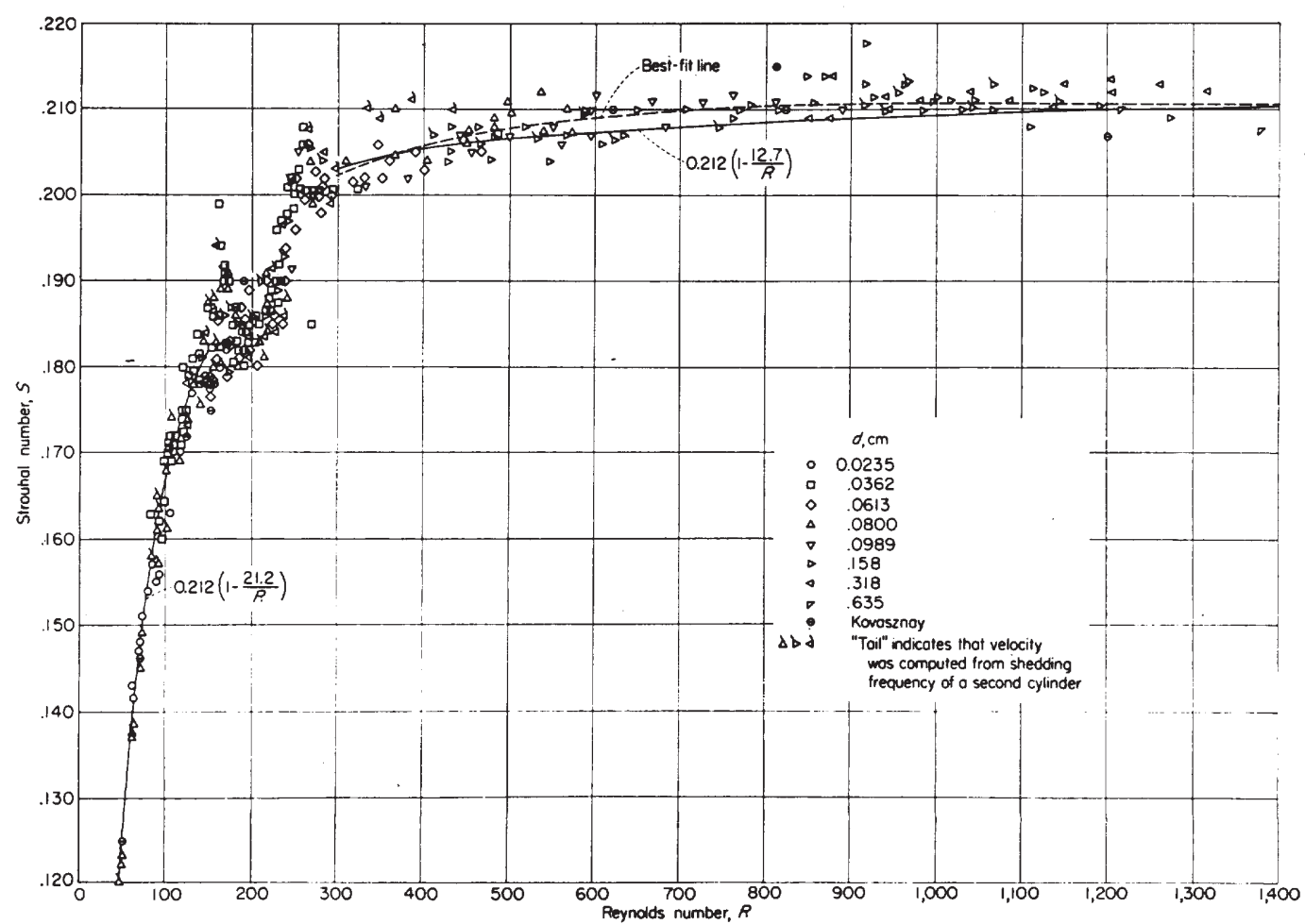

Figure 3: The Strouhal-Reynolds number relationship for $\operatorname{Re} \leq 1,400$. Taken from [22].

and measured Strouhal number could be as large as $40 \%$ for a circular cylinder. Given that vortex shedding and resonant frequency tuning is required for maximal energy harvesting potential, these mechanisms must be considered; especially when an EIE flutter system is studied in an environment where the time-averaged flow direction is nondeterministic, such as in the atmospheric wind (see section 4).

\section{Harvester types}

In the previous section, we have briefly reviewed two types of flutter that have been exploited for the purposes of energy harvesting. Here, we focus more on the harvester apparatus itself and the work done specifically in the field of flutter energy harvesting. It should be noted that there has been work examining both EIE-type and MIE-type harvesters [27].

\subsection{Common piezoelectric materials}

As shown in Eq. (5) in section 2.1, the critical flutter speed of an immersed beam varies as $\left(Y h^{3}\right)^{1 / 2}$, the effective

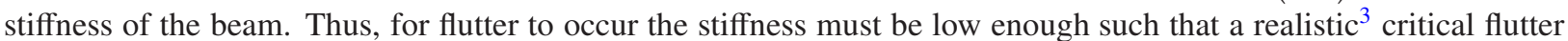
speed is obtained. This has led researchers to utilise compliant beams and in particular, compliant piezoelectric materials. Although there are many different types of piezoelectric materials, there have generally been three piezoelectric materials used in the field of flutter harvesting; namely, Lead Zirconate Titanate (PZT), PVDF, and Macro-Fibre Composite (MFC). Electromechanical properties of each of these materials varies with design and manufacturing processes, and it is not our intent to provide an exhaustive list of properties; rather, a brief and general overview of each material.

\footnotetext{
"Realistic", in this context, refers to critical flutter speeds that would be routinely encountered in an outdoor environment, where these harvesters are envisioned to be deployed. See section 4 for more details.
} 


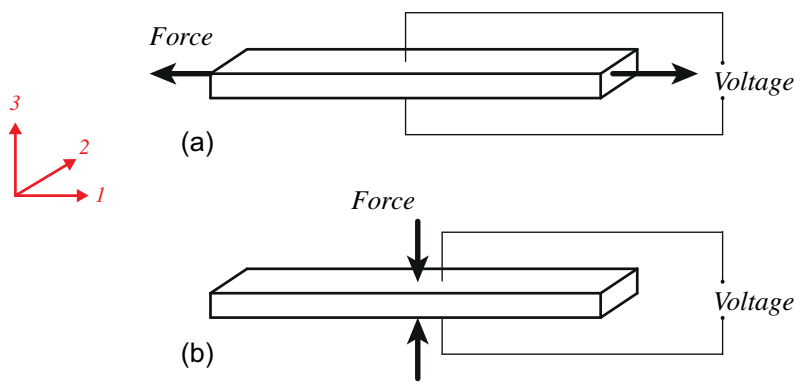

Figure 4: Schematic of (a) -31 and (b) -33 piezoelectric modes of operation.

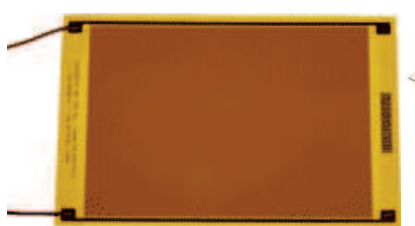

(a)

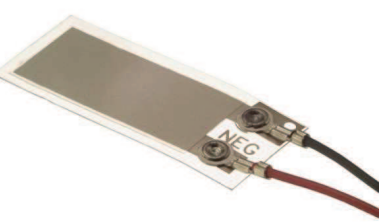

(b)

Figure 5: (a) Typical MFC [33] and (b) PVDF patches [34]

PZT was discovered in 1950, and since then has become the main piezoelectric ceramic used for a wide variety of dynamic sensing applications [28]. Although PZT is technically classed as a ceramic, with a high stiffness that would be unsuited for lower-frequency applications such as flutter harvesters, modern manufacturing techniques have allowed for PZT patches that are deployable in low-frequency energy harvesting applications (e.g. [29]). This has lead to the development of MFCs, which are generally comprised of precision engineered PZT fibres enclosed in adhesives, electrodes, and polyimide film [30], see Fig. 5a. MFCs are capable of relatively high power densities compared with other piezoelectric materials, with [31] reporting $P_{E A A}$ up to $440 \mu \mathrm{W} / \mathrm{cm}^{2}$; this is due to the relatively high piezoelectric coefficients, especially in the -31 bending mode (Fig. 4).

In 1969, it was found that PVDF exhibited strong piezoelectric properties and, due to lower costs and greater availability, have replaced PZT and MFCs in many sensory and battery applications [32], see Fig. 5b. PVDF must undergo a poling process, where the polymer is strained and placed within a strong poling [electric] field. Also, since PVDF is a polymer it can be engineered to suit many different stiffness and toughness requirements, giving a versatility advantage over PZT and MFCs. However, despite these advantages, PVDFs possess relatively low piezoelectric coefficients and by comparison, generally output an order of magnitude less power than an MFC of similar dimensions.

\subsection{Movement-Induced Excitation harvesters}

MIE flutter harvesting has been investigated at a fundamental level. A "flutter mill" was investigated by [35], which consisted of a cantilevered metallic beam oscillating between two magnetic panels thereby inducing an electric charge (Fig. 6). It was found there that the power outputs were comparable to a representative Horizontal Axis Wind Turbine (HAWT) at lower wind velocities, and exceeded HAWT outputs at higher wind velocities. The work in [35] was furthered by [36] and instead a cantilevered beam with a PZT bonded to the surface was examined (Fig. 7). Experiments agreed with theory relatively well and demonstrated that at a wind speed of $27 \mathrm{~m} / \mathrm{s}(\sim 100 \mathrm{~km} / \mathrm{h})$ a power per unit length of $870 \mathrm{~W} / \mathrm{m}$ could be attained. However, winds of this magnitude would rarely be encountered in outdoor conditions. In [37], a low-stiffness beam in a viscous flow was studied, similar to the study by [18], but for the purposes of energy harvesting. It was shown that the energy harvesting potential depends highly upon the piezoelectric layer thickness, coupling factor and resistivity of the piezoelectric circuit. In [38], a fluttering PVDF uni-morph was examined and a novel energy-capture circuit using a quasi-resonant rectifier was developed, which gave several times more power output than a conventional circuit using full-wave rectification.

One of the first concepts of a deployable flutter energy harvester was by [39], who envisioned a tree-like construct consisting of potentially hundreds of 'leaf-stalk'-type piezoelectric harvesters, see Fig. 8. This concept was realised 


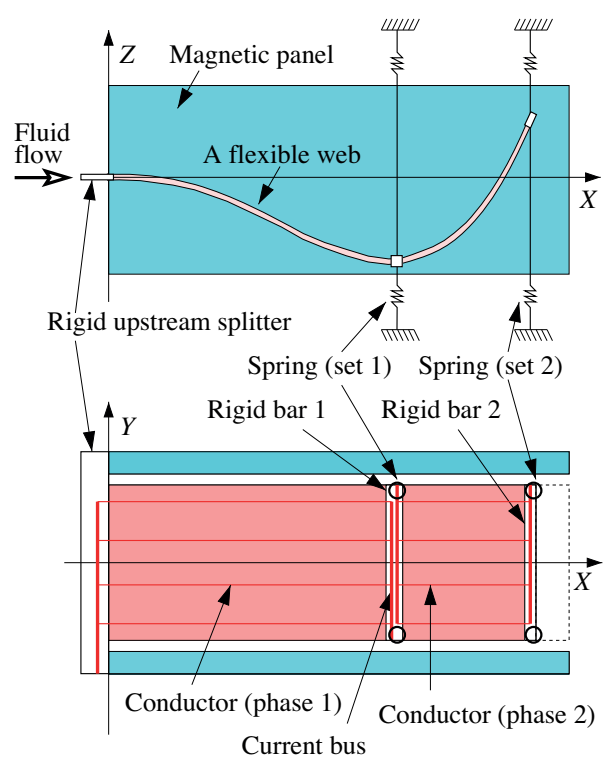

Figure 6: Flutter mill utilising a changing magnetic field for electrical current induction. Taken from [35].

in a study in smooth, parallel-flow conditions with a PVDF uni-morph stalk hinged to a polymeric leaf, and it was found that with a certain harvester configuration, $P_{E A A} \leq 45 \mu \mathrm{W} / \mathrm{cm}^{2}$ were attained [40, 41]. It was also noted that a triangularly shaped leaf caused the greatest power outputs amongst other geometric shapes, though no reasons were given as to why this was the case. Also, it was still unknown what the effects of a hinge had on the dynamics of the system, and so in [42] analytical, experimental and numerical studies were performed in smooth, parallel flow to determine the effects of a hinge, and its span-wise location. It was found that by introducing a hinge into the beam at half-span, the flutter frequency and critical flutter speed were reduced by nearly $50 \%$ compared to a uniform beam. Placing the hinge near the beam root at a vibration node gave similar behaviour to the uniform beam; placing the hinge near the beam free-end increased the system stability and caused flutter to occur in a higher order mode shape. The corollary of these results are that placement of the hinge along the energy harvester length could be used to tailor the harvester to a specific environment.

In [43] the aeroelastic parameters of their leaf-stalk flutter harvester (Fig. 10) were studied and it was discovered that by altering the leaf mass properties, significant differences in flutter behaviour would result. Similar findings were also reported in [44]; by changing the leaf geometric properties, the harvester could be tailored to different environments (i.e. with high or low prevailing wind speeds). This is important to consider since maximum power output is achieved when the harvester is in LCOs. Findings showed that the quantity of leaf area - not distribution (Fig. 9), affected the regime in which the harvester fluttered. Larger areas for a given wind speed tended the harvester to chaotic flutter with significantly reduced power output [44].

Work has also been done on understanding the aerodynamic proximity effects between multiple flutter harvesters. Work in $[46,47]$ involved flow visualisation on two of their harvesters placed in tandem, and it was observed that the trailing harvester would output up to $20 \%$ greater power than the leading harvesters, due to wake effects. Elsewhere in [48], 40\% greater output power from the trailing harvester was reported. The underlying mechanism governing this phenomenon was studied with flow visualisation and synchronised dual-camera setups (Fig. 11) to determine the specific cause of the power output increases $[49,50]$. It was found that the cause originated from the near-wake flow structures, shed from the leading harvester, increasing the PVDF tip velocity of the trailing harvester via phase-locked pressure gradients. The increase in tip velocity related to an increase in PVDF strain rate, increasing output voltage and [hence] power.

The generation of bending strain is essential to the transduction from mechanical to electrical energy in a piezoelectric material and generally the strain has resulted from pure bending. However, torsional strains may increase the total energy harvesting potential of flutter harvesters. Alternate harvester configurations were investigated by [51, 52] 


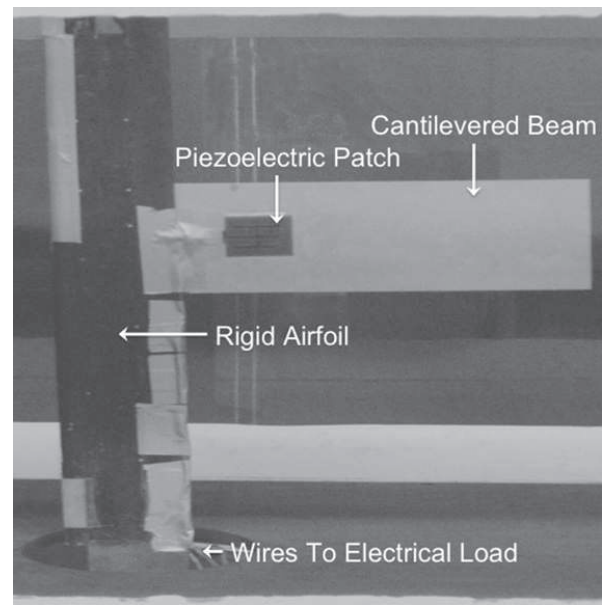

Figure 7: Flutter mill utilising a PZT patch bonded to a uniform beam. Taken from [36].

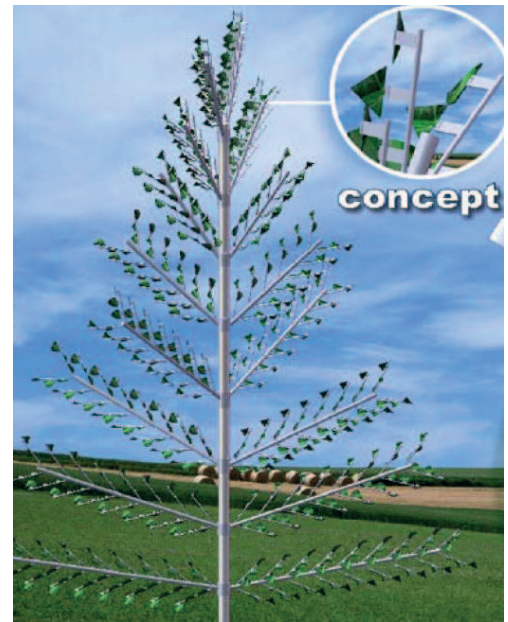

Figure 8: The piezoelectric tree concept. Taken from [39].

(Fig. 12b), whereby the flow would excite both torsional and bending modes of the harvester. Though previous studies stated that torsional modal excitation could add as much as $30 \%$ to total power output with base excitation [53], it was found that for their harvesters the flow-induced torsional excitation contributed several orders of magnitude less power output than the bending strains [51, 52]. Moreover, the alternate configuration output less power over the wind speed testing range than the conventional configuration because of the deviation from pure bending. A vertical-stalk configuration was tested in [40, 41] (Fig. 13) and it was discovered that due to the relatively large bending deformations compared to the conventional configuration, higher power outputs were attained.

\subsection{Extraneously-Induced Excitation harvesters}

One of the first studies into EIE flutter harvesting was by [7], where a compliant beam with PVDF patches bonded to the surface was placed in the near wake of a vortex shedding flat plate (Fig. 14). Though power outputs were not reported, it was discovered that in order to maximise power output the vortex shedding should lock in with a natural frequency of the harvester. Lock-in need not necessarily be with the fundamental vibration mode of the harvester; increases in power output were still realised when the vortex shedding was tuned to the second vibration mode of a leaf-stalk harvester [45]. [54] and [38] examined harvesters with similar geometry to the study by [7], and suggested that groupings of these harvesters could be capable of power densities greater than a conventional HAWT. [55, 56] 


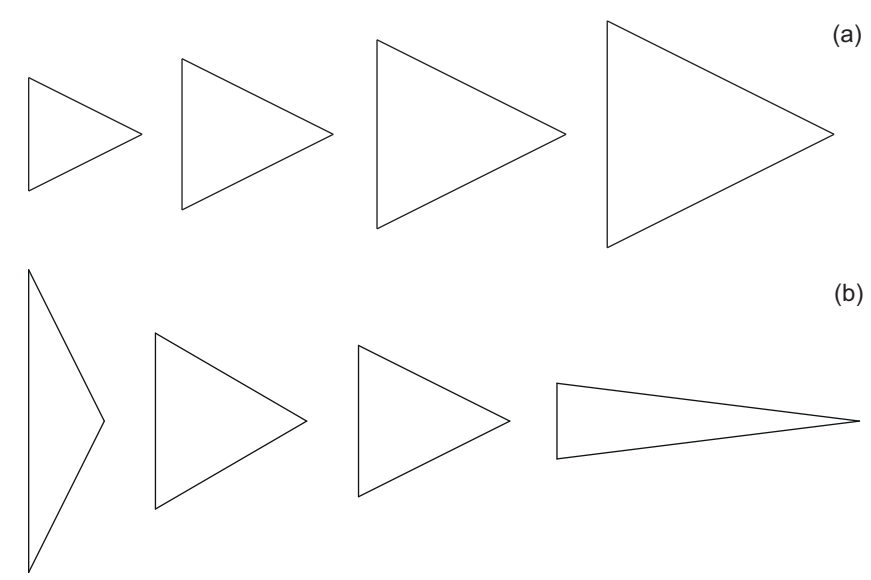

Figure 9: Differing leaf area (a) quantities with a fixed distribution, and (b) distributions with a fixed quantity. Taken from [45].

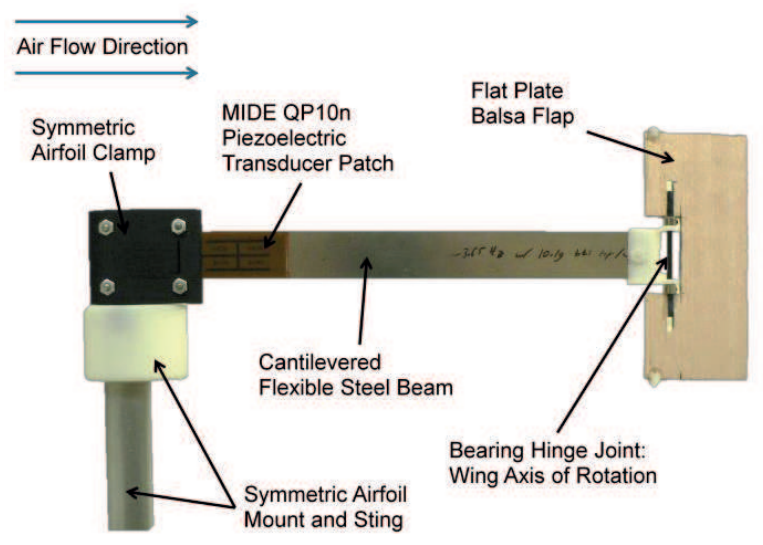

Figure 10: Leaf-stalk type harvester with PZT patch bonded to a thin steel beam. Taken from [46].

conducted PIV experiments and three-way, two dimensional aero-electro-mechanical simulations using coupled Computational Fluid Dynamics (CFD) simulations to determine outputs of a piezoelectric beam in the wake of a circular cylinder. The harvester was in a free-clamped configuration, as opposed to conventional clamped-free designs usually investigated, see Fig. 15. Even though the lift and drag coefficients obtained in the simulation were in relatively good agreement with findings elsewhere, two dimensional predictions of near-wake vorticity are known to be inaccurate due to the absence of modelling the three dimensional flow effects [e.g. 57-60]. Interestingly, [55] found that along the cylinder centreline in the wake, the power output of the piezoelectric beam dropped off as $(x / D)^{-3 / 2}$, where $x$ is the downstream distance of a piezoelectric beam and $D$ is cylinder diameter. This result was qualitatively consistent with work in [47] and [48-50]. Later work in [61] involved a vortex generator with a piezoelectric beam in a free-clamped configuration, which enabled controlled generation of vortices and permitted a detailed study of the fluid-structure interactions occurring. Two methodologies were presented by which the fluctuating pressure distribution over the piezoelectric beam, due to the vortices, could be determined. Utilising the PIV data, [61] employed both the control volume momentum balance method [see: 62] and direct integration of the pressure over the beam from the Navier-Stokes equations. The solutions from both methods agreed reasonably well.

A different approach to EIE harvesting was taken by [63], where circular cylinders were placed in tandem at varying spacings in a flow (Fig. 16). The cylinders were attached to piezoelectric disc transducers near the root and permitted to vibrate in the cross-stream direction only. Initial tests showed that power outputs were significantly lower than other harvesters, due to the size and quality of piezoelectric material selected. However, there were findings 


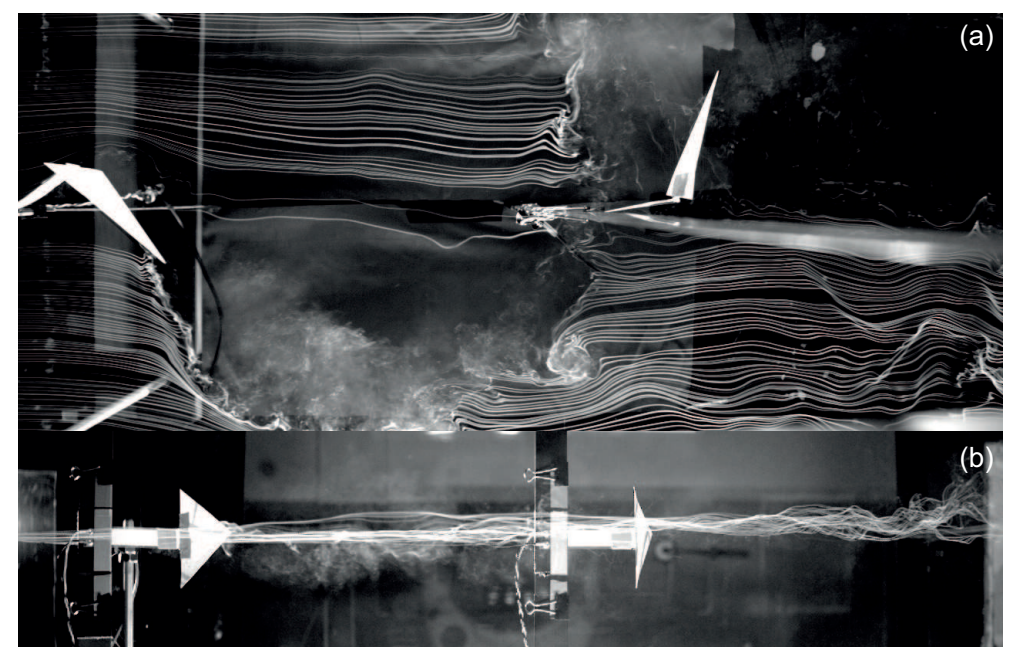

Figure 11: (a) Top- and (b) side-views of tandem-harvester flow-visualisation studies by [50].

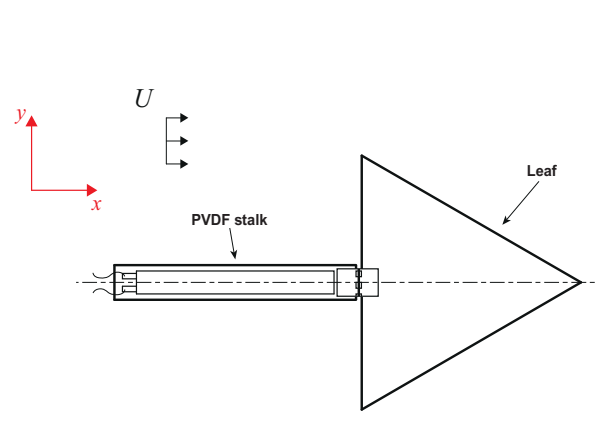

(a)

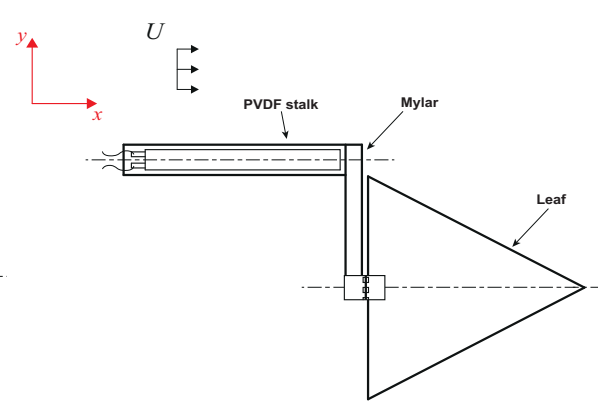

(b)

Figure 12: (a) Horizontal and (b) asymmetric harvester configurations studied in [51, 52].

similar to [47] and [50], in that there seemed to be an optimum tandem spacing between harvesters that caused trailing harvesters to output significantly more power than the leading one. This is in contrast to HAWTs, which are generally not placed in tandem configurations due to streamwise energy deficits in near-wakes [64]. [65] examined so-called "piezoelectric grass", where multiple PZT patches were arranged such that bending vibrations occurred in a bluffbody near-wake flow (Fig. 17). $1 \mathrm{~mW}$ per PZT cantilever was obtained at a flow velocity of around $11.5 \mathrm{~m} / \mathrm{s}$, and it was concluded that optimum turbulence conditions exist to maximise power outputs. Temporal and spatial turbulence scales were measured using custom built pressure probes, but the frequency response characteristics of these probes became undesirable above $300 \mathrm{~Hz}$, which is insufficient for high-fidelity turbulence measurements (e.g. see [66]).

\section{Wind characteristics and the Atmospheric Boundary Layer}

Almost all the research into energy harvesters has been carried out in smooth flow domains; either experimental or computational. EIE flutter harvesting thus far, though exploiting bluff-body vortex shedding, have been mostly in deterministic conditions with the dominant driving frequency being the vortex street excited in smooth upstream flow; thus effectively disregarding other sources of aerodynamic flow energy ${ }^{4}$. The majority of research thus far has been on harvesters that will potentially harness energy from the atmospheric wind and when placed in this flow environment,

${ }^{4}$ Refers to other sources of energy inherent in a flow that usually are ignored because the dominant [vortex] shedding mode is of key interest. 


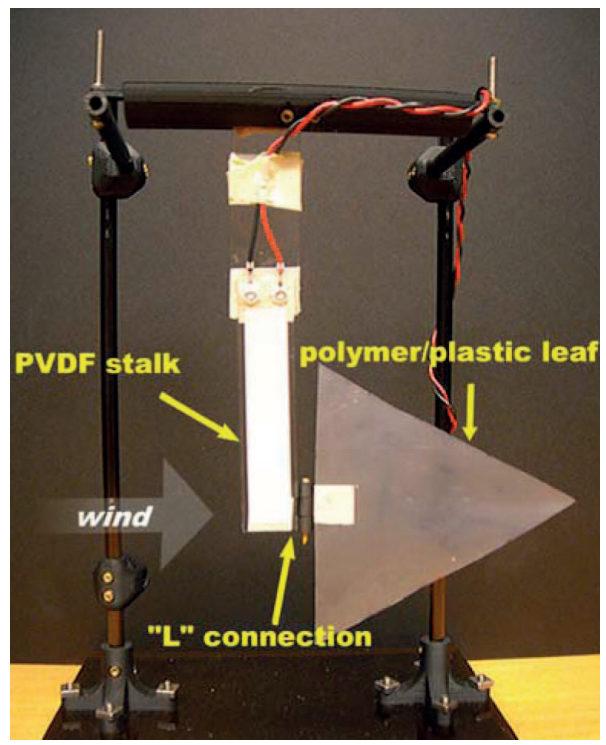

Figure 13: Vertical leaf-stalk harvester configuration studied by [40, 41].

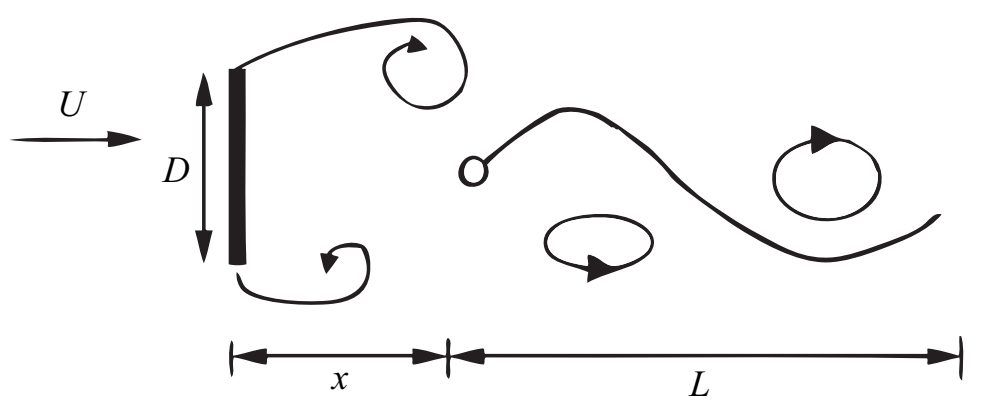

Figure 14: A beam with PVDF patches fluttering behind a flat plate. Image adapted from [7].

harvesters will be exposed to a flow that is much more complex. Assuming harvesters will be placed relatively close to the ground (in a similar manner to conventional rotary wind generators) they will be immersed in the lower levels of the Atmospheric Boundary Layer (ABL, sometimes termed the planetary boundary layer); a region where the wind are significantly influenced by the roughness of the ground.

\subsection{Characteristics of the $A B L$}

Mean wind speeds increase with height up to the Gradient Height, essentially the thickness of the ABL. This variation of velocity with height and the associated turbulence characteristics has been studied for many years, and is known to vary significantly with elevation and ground roughness [67], see Fig. 18. It is also influenced by the stability of the atmosphere (adiabatic, or various degrees of stability) [68], but this effect is not considered further here.

The relatively small size of fluttering energy harvesters means that it is likely that the time-averaged velocity gradient in the ABL can be ignored; however the turbulence levels at any point in space can be significant. Knowledge of how the flow environment is likely to vary in time and space - particularly at the scales applicable to energy harvesters - is useful for evaluation and optimisation of harvesters in either computational models or physical replications of the "real world" flows. Hence here we consider the turbulent flow environment in more detail.

The ABL has been documented by many workers and a large volume of work exists on understanding the turbulence inherent in atmospheric winds and its effects on the response of (essentially rigid) structures and aircraft; see for example [69, 70]. A recent book documents the last fifty years of wind engineering [71], including the possibilities 


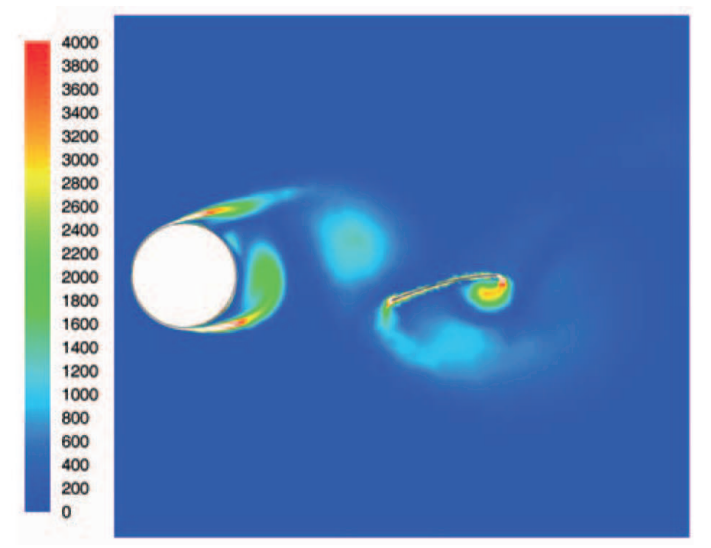

Figure 15: Two-dimensional aero-structural-electrical simulation of free-clamped harvester immersed in a vortex street. Taken from [56].

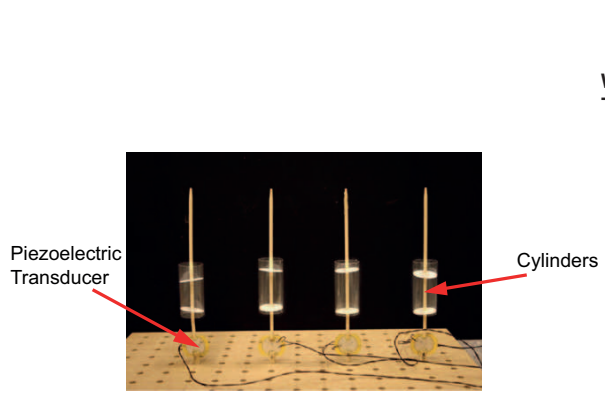

(a)

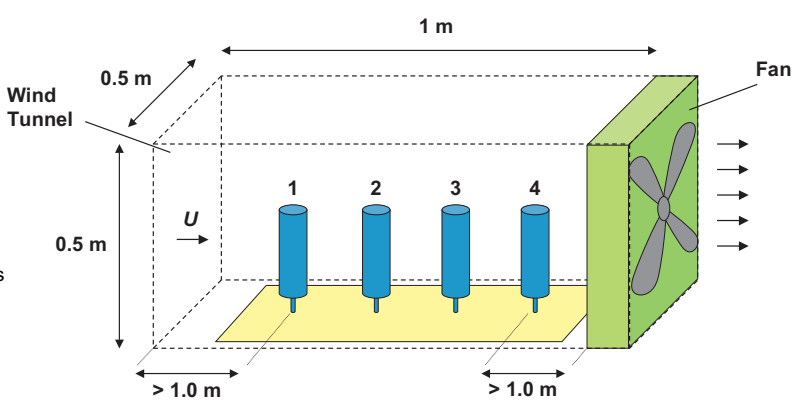

(b)

Figure 16: The (a) experimental specimens and (b) experimental setup of the tandem circular cylinders studied by [63].

of prediction via CFD. Information has been obtained by meteorologists and wind engineers using relatively large anemometers on fixed masts at heights ${ }^{5}$ well removed from the ground in order to predict loadings on masts, tall buildings, etc. [72-79].

It is useful to consider the likelihood of experiencing any given level of mean atmospheric wind. Whilst mean wind speeds vary with several factors, including; time of day, month of the year, location, elevation etc. (and details can be found from meteorological agencies for specific locations and times), an example is provided for illustrative purposes, see Fig. 19a. These data are for a coastal site and were obtained from an anemometer placed at $10 \mathrm{~m}$ from the ground. It can be seen that the most likely wind speed is of the order of $6 \mathrm{~m} / \mathrm{s}$ and the likelihood of experiencing calm is about 7\%. In Fig. 19b the variation of mean monthly wind speed is shown at an elevation of $10 \mathrm{~m}$ for the centre of a large city (Melbourne, Australia) and the average wind speed is about $11 \mathrm{~km} / \mathrm{h}(\sim 3 \mathrm{~m} / \mathrm{s})$. The city centre has a noticeably lower average wind speed than the coastal site, which is common for many parts of the world. The local wind conditions will be influenced by the specific location; including elevation effects (due to the average velocity profile), as well as possible over- or under-speed regions influenced by the wind effects of buildings or other obtrusions in the landscape. However these site-specific considerations are out of the scope of this review.

A compendium of many sources of data on the ABL can be found in [77] which summaries data up to 1974 and [78] revises and summarises single point data to 1985. A third data sheet, [79] details the variations in atmospheric turbulence in space and time for strong winds. A depiction of atmospheric turbulence is given in Fig. 20 (note the person standing in the left hand side for an indication of scale). Although surface tension effects minimise the influence of the extremely small structures in the atmosphere, distortion of the soap film depicts some of the small-to-medium

\footnotetext{
${ }^{5}$ Anemometers are generally placed well away from local influences at an elevation of $10 \mathrm{~m}$.
} 


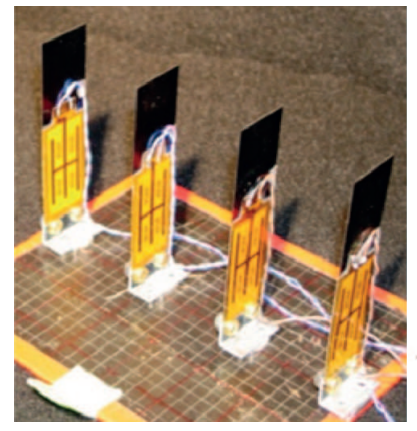

(a)

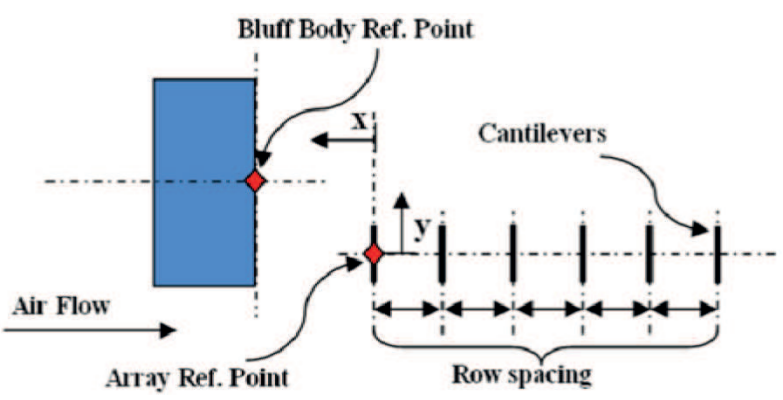

(b)

Figure 17: The (a) PZT patches and (b) plan view of the piezoelectric grass studied by [65].

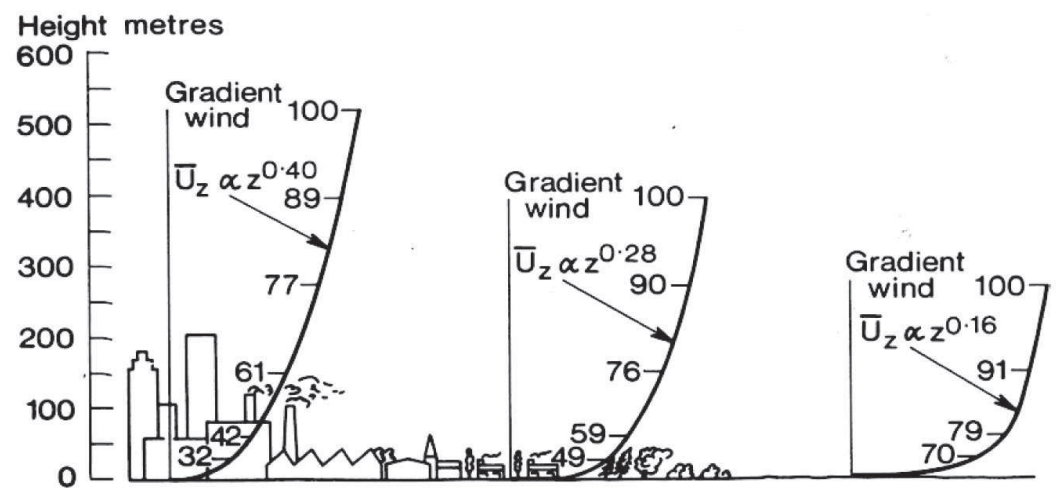

Figure 18: Mean wind profiles for a range of ground roughness, from [67].

scale structures in the first few metres of the ABL. The influence of various scale eddies are apparent, ranging from less than half a metre to approximately $15 \mathrm{~m}$ (the total length of the bubble is 32 metres).

Turbulence intensities for the three orthogonal velocities give a measure of relative gustiness in the atmosphere, and for the streamwise (along-wind) turbulence intensity;

$$
I_{u}=\frac{u_{R M S}^{\prime}}{\overline{R_{v}}}
$$

where $u_{R M S}^{\prime}$ is the root-mean-square of the fluctuating streamwise velocity and $\overline{R_{v}}=\overline{\sqrt{u^{2}+v^{2}+w^{2}}}$, the over-bar denoting mean quantites. Note that to obtain cross-stream or vertical turbulence intensities, $u^{\prime}$ is replaced with $v^{\prime}$ or $w^{\prime}$ respectively in Eq. (9). The mean velocities and intensities vary primarily with terrain and height from the ground. For very rough terrain, such as the city centre depicted on the left hand side of Fig. 18, intensities can reach 50\% close to the ground. [81] has measured the variations of intensities in the vertical direction up to about $20 \mathrm{~m}$ for smoother "suburban" terrain, such as shown in the centre of Fig. 18. From such measurements it can be seen that with increasing closeness to the ground the turbulence intensity increases and changes characteristics (Fig. 21). As the ground surface is approached the vertical fluctuations are attenuated, thus turbulent energy is mainly in the horizontal plane. However, there can still be significant energy in all directions in the last few metres of the Earth's surface. It is clear that when the harvesters are placed in any terrain within the ABL, the upstream flow will be far from smooth.

The spectra of the three orthogonal velocity components provide descriptions of the frequency contents. Whilst there is a wealth of data from wind engineering and meteorological measurements on this topic the focus has been on strong winds at higher elevations under conditions of neutral thermal stability. Note that spectra can be presented in dimensionless or dimensional forms (the latter will be used here) where the latter has units of $(\mathrm{m} / \mathrm{s})^{2} / \mathrm{Hz}$. 


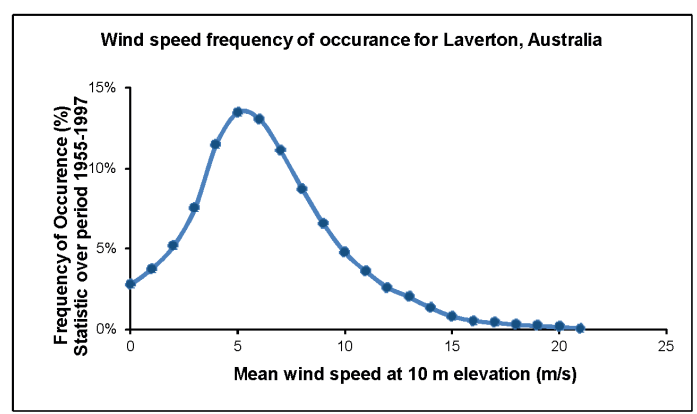

(a)

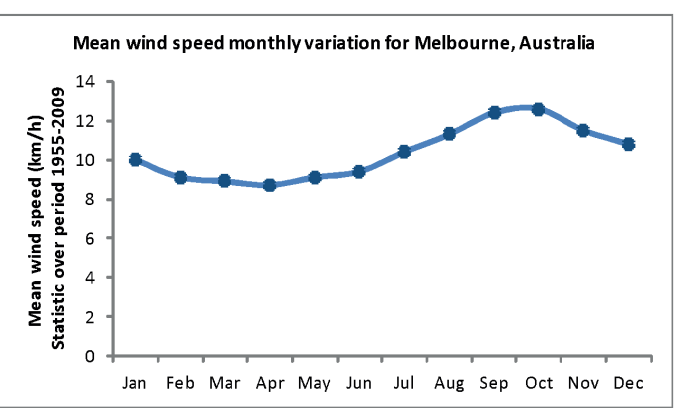

(b)

Figure 19: Representative wind statistics from [80] showing (a) probability of occurrence and (b) typical monthly variations for two different sites. Note the difference in velocity units.

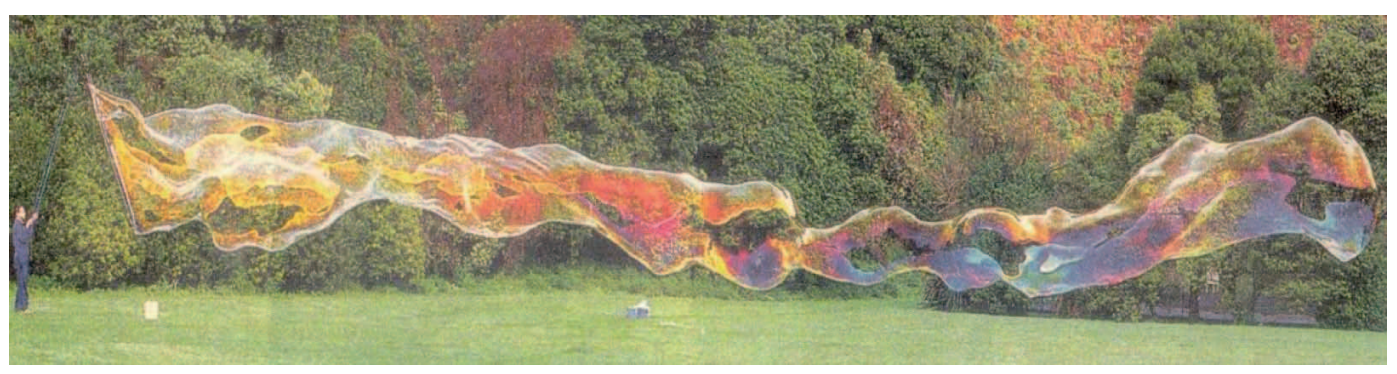

Figure 20: The longest bubble in the world. (Image courtesy of Alan McKay, copyright Garry Norman).

A dimensional energy spectrum of the streamwise turbulence measured during relatively strong winds encompassing a very wide frequency range is shown in Fig. 22. Data were obtained via anemometers located in "very rough" terrain at approximately $100 \mathrm{~m}$ from the ground. Whilst these data are for a specific location, [74] noted that the spectra from other locations exhibit similar spectral gaps and amplitudes. Four distinct peaks can be seen in the spectrum. They include three peaks at very low frequencies, which could be considered as quasi-static in terms of energy harvester dynamics. Of most relevance is the peak at the right hand end. It is separated from the longer-term "weather map" peaks by a gap centred at a period of one hour, suggesting that a one-hour average will capture turbulence effects in the ABL well and will exclude the longer-term influences. Since the scale of energy harvesters is small it is likely that we are only interested in time scales that are shorter than a few tens of seconds thus the most influential energy will be in the so-called micrometeorological range.

The data shown in Fig. 21 (and for the data compiled in [77-79]) were obtained with averaging periods of typically 20-60 minutes and are high-pass filtered to eliminate the very low frequency components resulting from "weathermap" fluctuations. This was done on the basis that the influence of large scale, low frequency fluctuations in the wind can be considered as quasi-static on buildings and structures. Clearly this will also be the cases for smaller objects such as energy harvesters. From Fig. 21 and data given in [77-79] it can be seen that the range of streamwise turbulence intensity is between 15 to $20 \%$ reducing with elevation up to $20 \mathrm{~m}$. However this also includes scales of turbulence that are considerably larger than the harvesters (but still significant for a building). Thus a very wide range of intensities and scales can be experienced in the ABL, depending upon terrain and elevation. When considering replication of this turbulent environment the selected intensity will depend upon at which frequency one decides to consider as quasi-static.

\subsection{Flutter harvesting in ABL environment}

The flow direction, relative to the ground, can vary in the range $0^{\circ}$ to $360^{\circ}$. Thus the wind may also approach the harvester from any direction in the outdoor environment. 


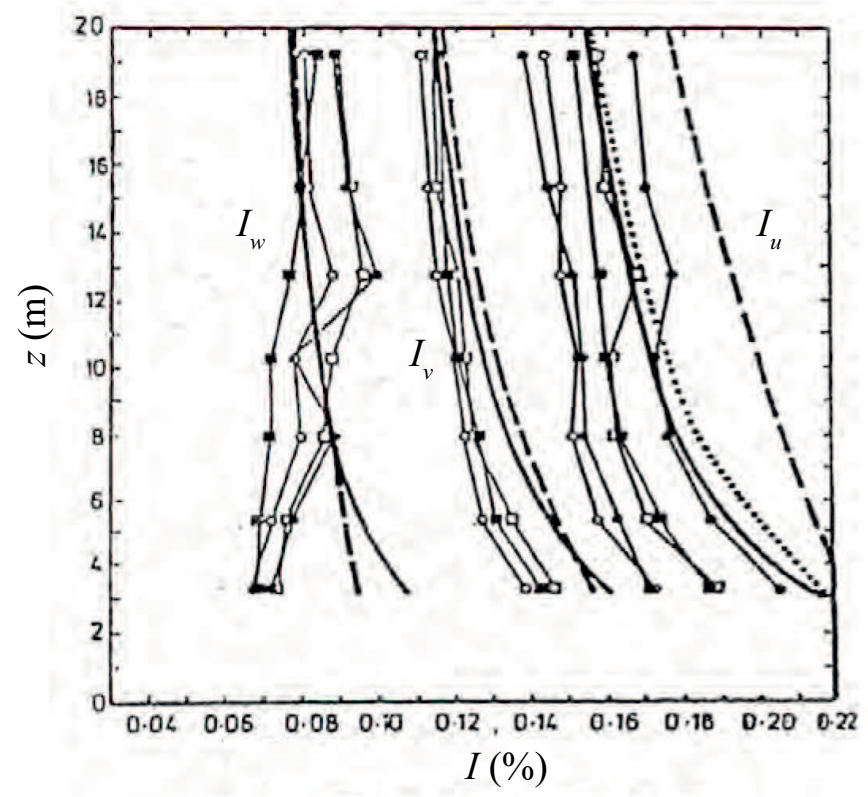

Figure 21: Turbulence intensity profiles for "suburban terrain", from [81].

Work by $[45,82,83]$ involved investigation of the behaviour of a leaf-stalk harvester exploiting EIE flutter in a replicated ABL flow. Though EIE flutter could only be exploited for parallel-flow conditions, the output power from the harvester was compared in smooth and turbulent flows with varying wind speeds and direction (Fig. 23). Aspects of the ABL were replicated by using turbulence-generation grids mounted in the inlet of an industrial wind tunnel. Only length and temporal scales relevant to the harvester size were generated, since generating the entire spectra of the ABL is infeasible. The experimental setup is shown in Fig. 24. The turbulence intensity selected was $12.7 \%-$ relatively low when compared to intensities encountered in the ABL; however, this percentage does not include the low frequency energy associated with the larger turbulence scales in the atmosphere. That is, this $12.7 \%$ intensity is only the high frequency part of the ABL spectrum; thus, it may be considered as the most relevant part of the relatively broad micrometeorological spectrum.

Results showed that regardless of smooth or turbulent flow, $P_{E A A}$ from the harvester decreased significantly given off-axis winds. However, slightly higher power outputs were reported from the harvester at highly off-axis flow directions in turbulence at higher wind speeds, due to the energy of fluctuations exciting higher-order vibration modes of the harvester. It is clear that for off-axis winds, turbulence provided a marginal advantage over smooth flow at higher wind speeds. Conversely, for parallel flow turbulence was detrimental to harvester performance. These observations may be seen in Figs. 25 and 26, where the yaw angle results are compared for smooth and turbulent flow, respectively.

\section{Embodiment and optimisation}

\subsection{Flutter harvester system}

There are other areas in flutter harvesting other than just the flutter harvester itself. The voltage generated by a flutter harvester may be used readily for charging a battery, or for immediate use in powering a ULP device. Generally, it is not feasible to utilise the voltage straight from the harvester - it must be conditioned through a circuit system. Thus, the flutter harvester and energy capture circuitry could be considered as a flutter harvesting system. There have been studies that have examined the system as a whole, or solely the energy capture circuitry. [84] conducted a similar investigation to [7], with an energy harvesting "eel" placed in the wake of a vortex shedding bluff body. However, the focus of their study was more on electrical subsystem optimisation, rather than flutter dynamics. [84] reported the use of a switched resonant-power conversion, which could overcome a number of difficulties with low-frequency voltage 


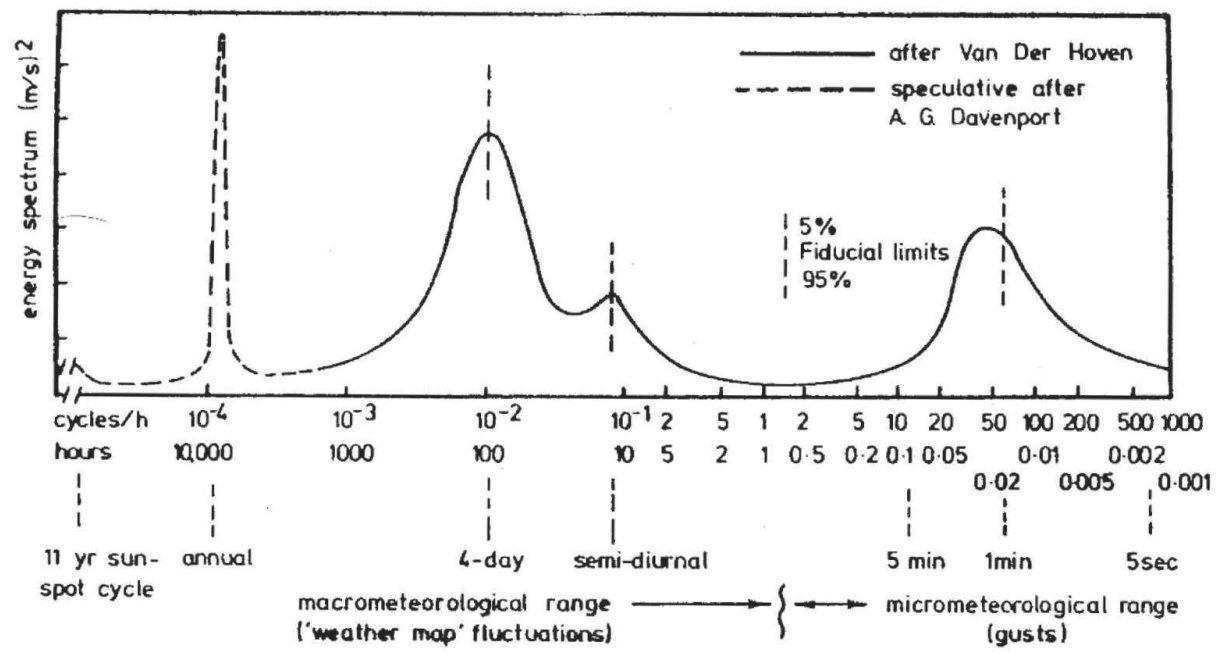

Figure 22: Spectrum of turbulence at $100 \mathrm{~m}$ from [74].

generation from a PVDF (Fig. 27). It was found that using this type of circuit the electromechanical conversion efficiency of the PVDF reached a maximum of $37 \%{ }^{6}$. [38] also implemented a similar circuit design to that of [84], called a quasi-resonant rectifier circuit, used for DC applications. The quasi-resonant rectifier circuit caused higher outputs than conventional full-wave rectification (Fig. 28).

An important aspect of flutter harvesting is the dependence of the output power with resistive loading in the circuit. It is well known that the power output from an AC voltage source is given as;

$$
P_{\text {mean }}=\frac{V_{R M S}^{2}}{R_{L}},
$$

where $V_{R M S}$ is the root-mean-square voltage and $R_{L}$ is the load resistance. [85] showed that the optimum load resistance may be approximated as;

$$
R_{L_{o p t}} \approx \frac{1}{\omega C}
$$

where $\omega$ is the oscillation frequency, and $C$ is the piezoelectric capacitance. Furthermore, it was seen in Eq. (4) (p. 4) that the flutter frequency depends linearly on the free-stream flow speed, $\omega \propto U$. [14] then showed that;

$$
\omega=k \sqrt{\frac{\rho_{f} U^{2}}{\rho_{s} h L}}
$$

where $k$ is some material dependent constant. By substituting Eq. (12) into Eq. (11) and subsequently into Eq. (10) the parameters on which maximum power output depend can be seen as;

$$
P_{\text {mean } n_{\max }} \approx V_{R M S}^{2} k C \sqrt{\frac{\rho_{f} U^{2}}{\rho_{s} h L}} .
$$

\footnotetext{
${ }^{6}$ Note that this is not the transduction efficiency from fluid kinetic energy to electrical energy through flutter of the piezoelectric material, but rather the efficiency of the conversion from mechanical strain energy to electrical energy.
} 


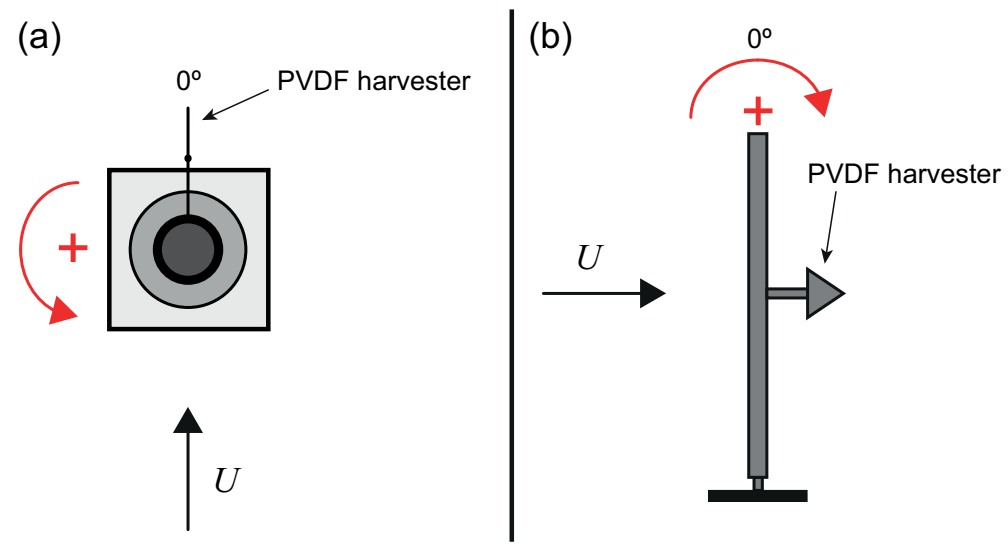

Figure 23: Sign convention for (a) yaw angle and (b) pitch angle. Taken from [45].

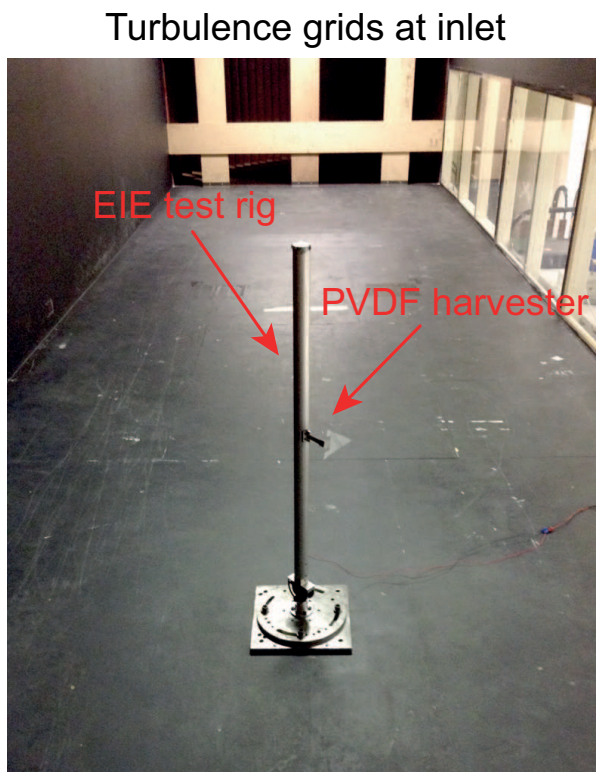

Figure 24: Photograph of the EIE rig installed in an industrial wind tunnel. Adapted from [45]. 


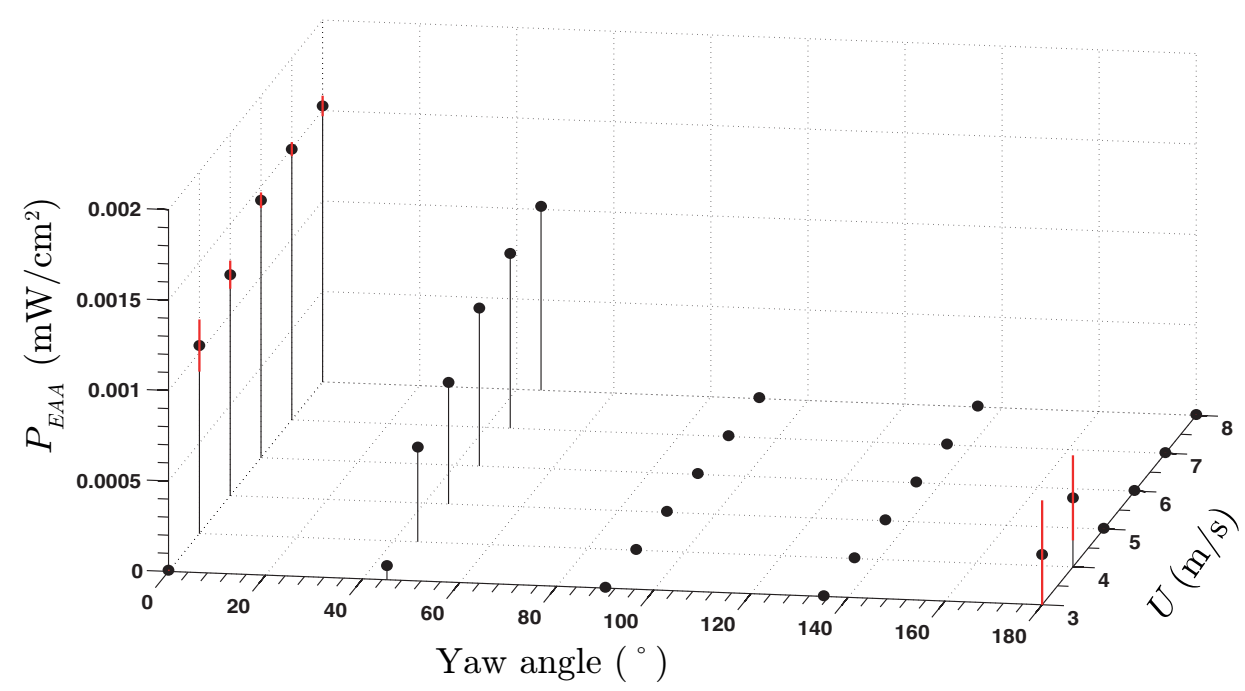

Figure 25: $P_{E A A}$ versus yaw angle and wind speed in smooth flow, with zero applied pitch angle. Uncertainty at $95 \%$ confidence is denoted by vertical red bars on the 0 and $180^{\circ}$ cases. Adapted from [45].

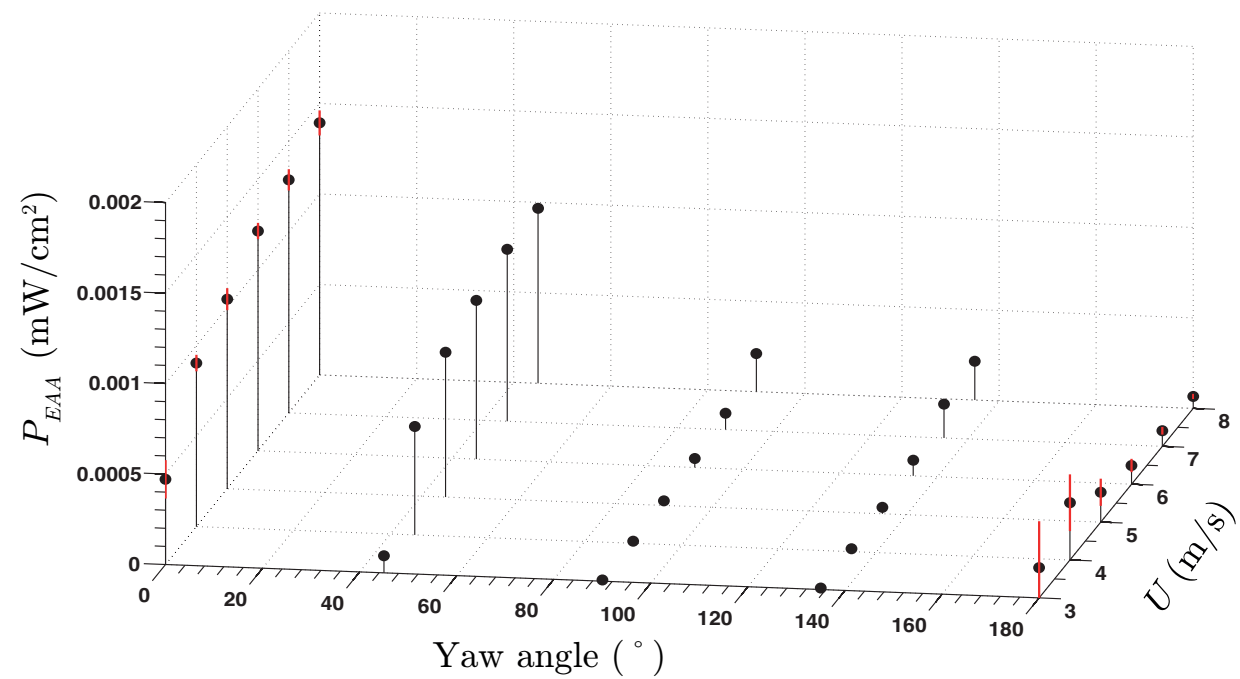

Figure 26: $P_{E A A}$ versus yaw angle and wind speed in turbulent flow, with zero applied pitch angle. Uncertainty at $95 \%$ confidence is denoted by vertical red bars on the 0 and $180^{\circ}$ cases. Adapted from [45]. 


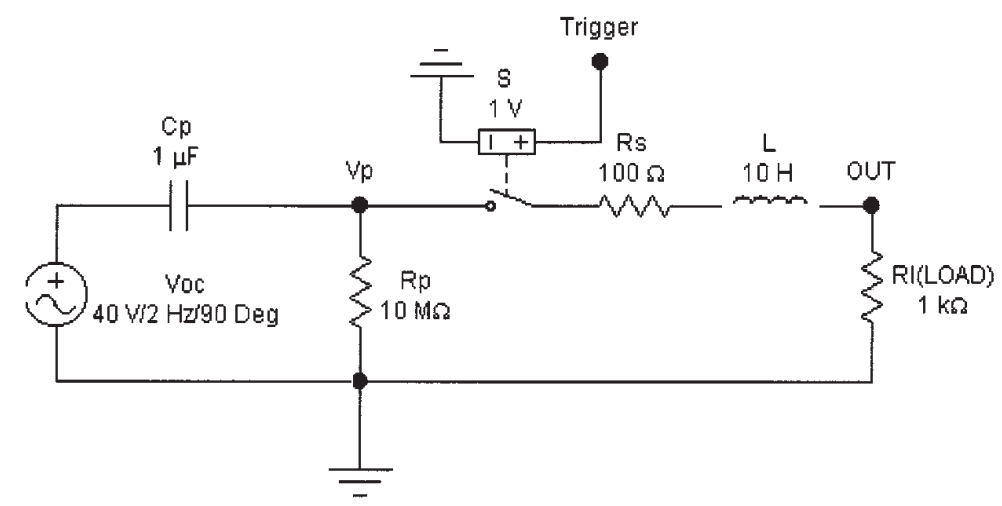

Figure 27: Switched resonant-power circuit used by [84].

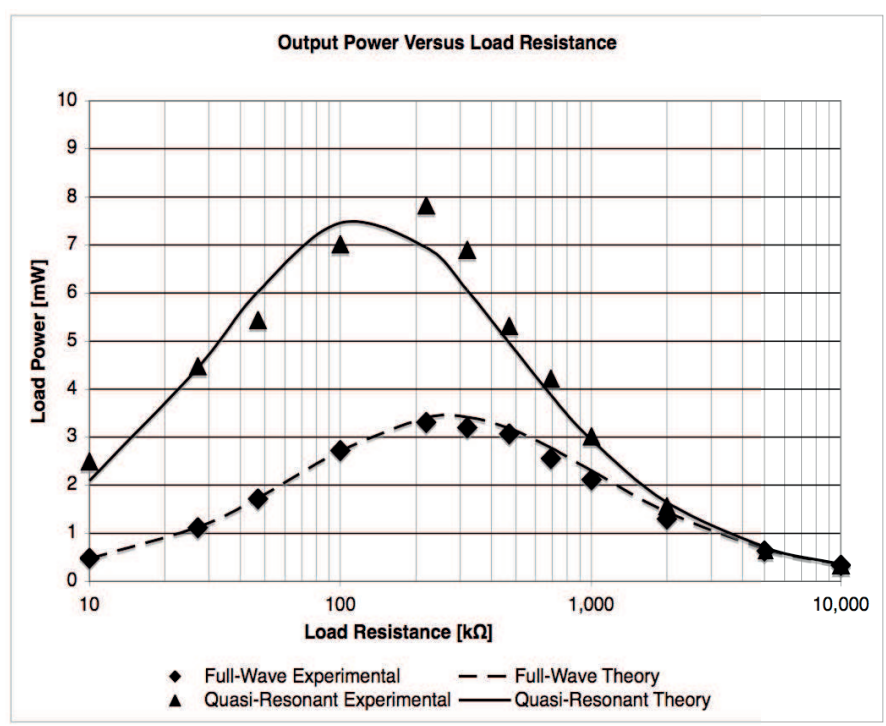

Figure 28: Comparison of full-wave and quasi-resonant rectifier circuits in [38].

There has been previous work on developing an active, self-powered resistance tuning mechanisms for flutter harvesters, but [86] proved that self-tuning never results in a net positive power output. Therefore, most studies simply tuned the resistance to a certain flow speed (or vibration frequency) of interest (e.g. see the review by [87]). A few studies involved a range of wind speeds and so the resistance was tuned to the median wind speed [45, 88]; however in a environment where the wind speeds are only quantified via statistical data, it would be more effective to tune the resistance to the mode wind speed.

\subsection{Performance metrics}

The determination of a robust method by which to quantify a flutter harvester's performance is of great importance. However, there have been varied, and sometimes contradicting, methods by which flutter harvester performance has been quantified.

It is known that the power contained in a fluid flow is given by;

$$
P_{A}=\frac{1}{2} \rho_{f} A_{\text {swept }} U^{3},
$$


where $P_{A}$ is the power available in the fluid flow and $A_{\text {swept }}$ is the cross-stream area "occupied" by the generator, such as a swept area disc of an axial rotary wind turbine, or the rectangular area "seen" by the flow approaching a Vertical Axis Wind Turbine (VAWT). Upon consideration of an unbounded flow, the well known Betz limit applies [89]; only a maximum of $59.3 \%$ of total energy in the wind may be extracted by a turbine rotor across an area. Most modern wind turbines are only capable of extracting around $40 \%$ of the total energy available in the wind. Thus, there are two important issues to be addressed with regards to the potential efficiency of flutter harvesters:

1. Does the Betz limit (or a similar fundamental principle) apply to flutter harvesters, and

2. How may the performance of a flutter harvester be effectively quantified in a range of flow conditions?

From fundamental considerations it would appear that the Betz limit applies and [90] stated that flutter harvesting indeed should be subject to the Betz limit. [35] studied the energy transfer between the flutter harvester and the surrounding fluid and discovered that at different span-wise locations along the beam length, energy would either be pumped in to the beam to sustain flutter motions, or energy would be pumped from the beam into the surrounding fluid. However, no concrete method by which to calculate flutter-mill efficiency was presented and comparison to existing HAWTs involved assuming an identical wind-receiving area, $A_{\text {swept }}$. Both [36] and [37] cited that the "capture" efficiency - the efficiency of converting flow energy to strain energy, depended on $A_{\text {swept }}=2 b q_{\text {max }}$, where $b$ is the beam width and $q_{\max }$ is the maximum theoretical amplitude of the beam during LCOs. [36] derived an expression for the capture efficiency $\eta_{C}$ in terms of the power delivered by the flow to the beam $P_{B}$ and the power in the flow $P_{A}$, as Eq. (15);

$$
\eta_{C}=\frac{P_{B}}{P_{A}} \approx \frac{\frac{1}{2} m_{p} \omega \int_{0}^{L}\left(\frac{\tilde{q}_{\max }}{\phi^{t p}} L \phi_{2} \omega\right)^{2} \mathrm{~d} x}{\rho_{f} U^{3}\left(b q_{\max }\right)},
$$

where $m_{p}$ is the mass per unit length of the beam, $\tilde{q}_{\text {max }}$ is the maximum experimental normalised beam tip displacement, $\phi^{t i p}$ is the maximum tip displacement of the normalised mode shape, and $\phi_{2}$ is the normalised mode shape. With this metric, the flow-to-structural energy conversion efficiency of the harvester studied by [36] was calculated to be just over $17 \%$ over a relatively wide band of wind speeds, while [37] reported a capture efficiency of around $10.6 \%$. Note that these efficiencies do not include the efficiency of the electrical subsystem and thus actual efficiencies would be lower.

A simpler, implicit approach was suggested by [84], who stated that;

$$
P=\frac{1}{2} \eta_{1} \eta_{2} \eta_{3} A_{\text {swept }} \rho_{f} U^{3}
$$

where $P$ is the harvester power output, $\eta_{1}, \eta_{2}$ and $\eta_{3}$ are the hydrodynamic (i.e. flow-to structural energy), electromechanical, and electrical (i.e. electrical subsystem) efficiencies respectively.

Other approaches to defining flutter harvester performance metrics have been taken. Instead of dealing with the efficiency, normalised power outputs have been reported. Power or energy densities have been defined in a few different ways, as previously mentioned in section 1.1. [40] defined a power output per unit area or per unit volume of the entire harvester, while others have cited power densities in terms of power per unit area of electro-active material only [45]. Table 1 details different power density calculation methods for studies where raw data were available; it is evident that just by changing the method by which the performance metric is calculated, different results may be reported.

[90] defined power density as the power output per unit swept area of the harvester, and gave a brief review of the efficiencies of other piezoelectric harvesters (Fig. 29). The efficiency isolines are given by the function;

$$
\frac{P}{A_{\text {swept }}}=\frac{1}{2} \rho_{f} U^{3} \eta_{\text {Betz }} \eta_{\text {iso }},
$$

where $\eta_{\text {Betz }}=0.593$ is the Betz limit, and $\eta_{\text {iso }}=[0.0001,0.001,0.01,0.1,1]$ are the isolines. Interestingly, the efficiency of the harvester reported in [36] (17\%) seems to contradict the value reported in [90] $(<0.01 \%)$, presumably 


\begin{tabular}{lcccc}
\hline Device & $P_{\text {EAA }}\left(\mathrm{mW} / \mathrm{cm}^{2}\right)$ & $P_{\text {Volume }}\left(\mathrm{mW} / \mathrm{cm}^{3}\right)$ & $U(\mathrm{~m} / \mathrm{s})$ & $R_{L}(\mathrm{M} \Omega)$ \\
\hline [40]'s short vertical stalk & 0.045 & 0.31 & 8 & 10 \\
[92]'s small windmill ${ }^{a}$ & 0.012 & 0.0051 & 4.5 & 0.02 \\
[46]'s harvester & 0.12 & 0.096 & 7 & Actively tuned \\
\hline
\end{tabular}

${ }^{a_{\text {The }}}$ windmill used consisted of 18 piezoelectric bi-morphs, where the bi-morphs consist of two uni-morphs bonded together. Thus, the total electro-active area was the length $\times$ width of one bi-morph multiplied by a factor of 36 .

Table 1: Comparison of piezoelectric harvester power output per electro-active area and per total device volume.

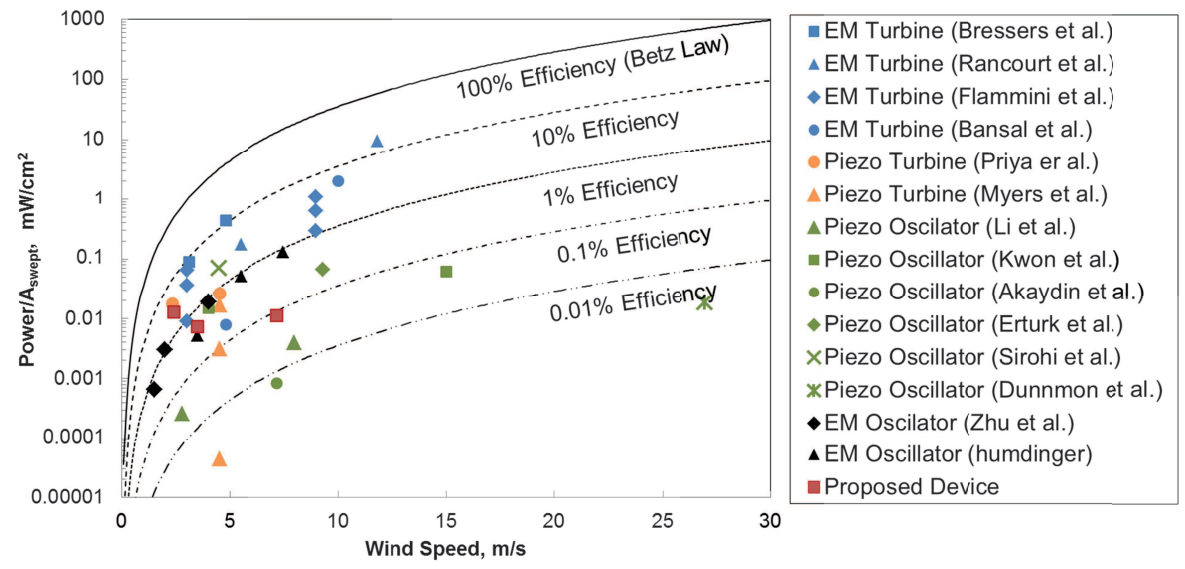

Figure 29: A comparison of different harvester performances relative to the Betz limit by [90]. Note that the EM and piezoelectric turbines are not actual flutter (oscillator) energy harvesters, but have been included for comparison.

due to the differing methods of determining harvester efficiency. It is postulated, but not conclusively stated, that the efficiencies reported using the method in [90] would change if, per say, Eq. (15) were utilised instead. Other workers disregarded the Betz limit altogether in their efficiency calculations [27]. To date, there is still a lack of a standardised method by which the efficiency of a fluttering harvester may be evaluated. This is a relatively major concern and must be addressed fundamentally in forthcoming work.

While it may not be possible to compare efficiencies between turbine (i.e. rotational) type and flutter harvesters yet, it can be seen from Fig. 29 that by normalising the output power by the swept area - the disc swept by the blades in a turbine, and [ostensibly] the cross-stream area occupied by the oscillator harvester, oscillator harvesters are not far behind turbines. This is especially true for the electromagnetic (EM) flutter harvesters, and the piezoelectric harvester by [91] that exploited aerodynamic galloping, not flutter.

\section{Concluding remarks}

Aside from the ultra low power densities and potential long Return On Investments (ROIs), one of the greatest challenges for flutter harvesting appears to be overcoming quantification and alleviation of fatigue damage. Flutter has historically been considered an adverse phenomenon because of the cyclic stresses and strains that occur in the structure as a result. Most modern aerodynamic or hydrodynamic designs are such that component flutter is avoided when operating within the design envelope of the vehicle (or device). Even so, stress-cycle $(S-n)$ curves are known for most metallic and composite materials prevalent in industry today. A gap in research exists in determining the fatigue life of a piezoelectric flutter harvester. Though a review of the few studies done in this area is outside the scope of the review here, it is quickly becoming necessary to determine the fatigue behaviour of flutter harvesters. It is highly likely that performance degradation occurs over time - the prevailing question is, how much time? PVDF damage was documented qualitatively by [45] after completing the study (Fig. 30), but no fatigue metrics were suggested.

It is also important that a performance metric for flutter-harvester evaluation must be set in place as interest grows. Without a standard performance metric, cost-effectiveness and ROI are unknowns and investment risk is high. There 


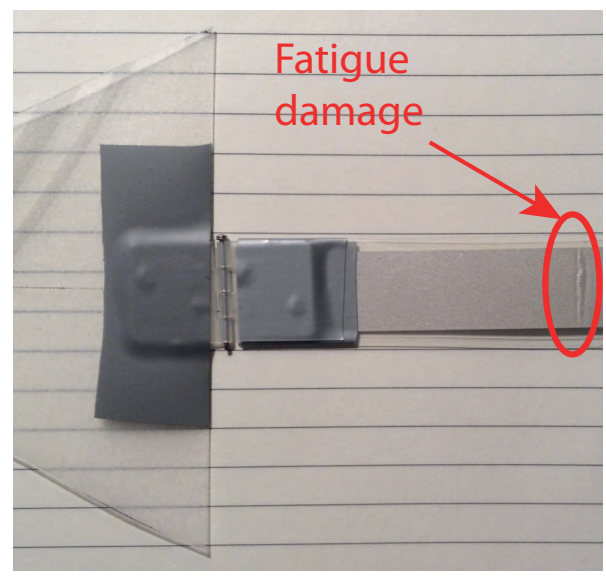

Figure 30: Suspected superficial fatigue damage of the harvester in the study by [45].

are many documented cases of flutter harvesting in an idealised (i.e. smooth, unidirectional) flow, and it could be that ROI is based on harvester performance in these ideal conditions, which will be extended when compared to the realistic outdoors. To the author's knowledge, other factors such as weather, have not been considered anywhere in the literature. Precipitation could have an added-mass effect on a flutter harvester which could alter harvester performance.

Nevertheless, piezoelectric flutter harvesting has increased in attractiveness and potential, due to the requirement for alternative methods to scavenge energy in quiescent environments. In [93] it was stated that there is a move of Ultra-Low Power (ULP) technologies ${ }^{7}$ from the conceptual state to the deployable design space. Examples of such technologies include wireless sensor nodes or certain types of LED lights. Given that some piezoelectric flutter systems possess electro-active area-based power densities as high as $440 \mu \mathrm{W} / \mathrm{cm}^{2}$ [31], a piezoelectric flutterbased system could potentially provide a cost-effective, off-grid solution to powering ULP technologies, in contrast to smaller-scale wind turbines as covered in section 1. Compliant piezoelectric materials continue to decrease in price (prices vary widely with dimensions but recently have been reported to be as low as $1 \notin / \mathrm{cm}^{2}$ (USD) for PVDF films that are un-poled ${ }^{8}$ [94]) and flutter systems may become comparable to existing renewable-energy technologies. For example, Photo-Voltaic (PV) technology was recently stated to cost as low as $2.2 \phi / \mathrm{cm}^{2}$ (USD) for a commercial silicon module with an efficiency of $16 \%$ [95].

There are other advantages to a piezoelectric flutter harvester system, in that such a system would potentially be quieter and possibly safer than a small-scale wind turbine. While a flutter system is likely to be much more susceptible to fatigue than a wind turbine, structural failure is less likely to cause any significant property damage or harm to bystanders.

In general, conventional wind turbines are not placed in tandem due to adverse wake effects, which cause problems in trailing turbines (loss of power, turbulence-induced vibration, gearbox resonance, etc.), see [64]. However, flutter harvesters under certain conditions appear to perform beneficially when placed in tandem. The ability to potentially extract more power from a trailing harvester in the wake of another harvester is attractive; however, the benefit decays after three or four harvester-lengths downstream [47]. This phenomenon could allow for more space-efficient designs compared with existing wind farm layouts. This is especially useful in highly urban environments, where space is considered a commodity. The matrix-like flutter harvester design initially proposed by [54], shown in Fig. 31, could become a reality now that harvester proximity effects are more fully understood [e.g. 50, 96]. However such an embodiment would need the harvester system to be aligned with the flow direction, which will not be the case for fixed systems in the ABL.

${ }^{7}$ ULP technologies are devices that consume low amounts of power - in the milli- or microwatt range.

${ }^{8}$ Refers to PVDF films that have not been poled through an electrostatic field and hence do not have piezoelectric properties. 


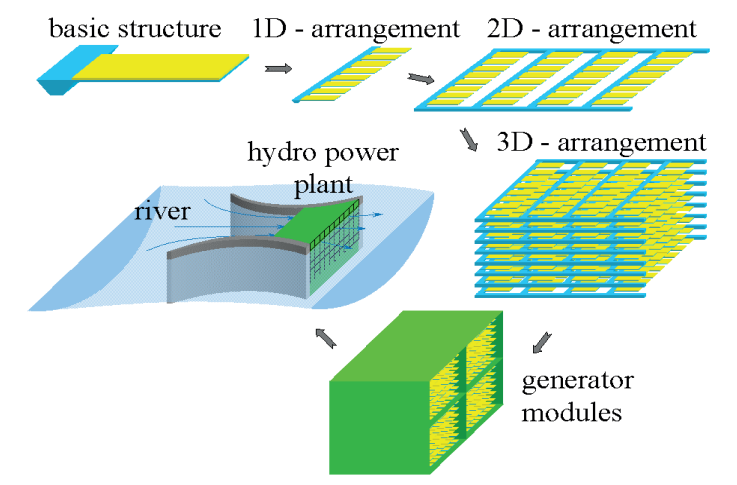

Figure 31: A matrix-like flutter harvesting device proposed by [54].

\section{Acknowledgements}

This work was funded by the Australian Research Council (ARC) grant no. LP100200034. The Partner Organisation for this project is FCST Pty Ltd. The author would also like to thank the Platform Technologies Research Institute at RMIT University for providing the necessary facilities for the review conducted here.

\section{References}

[1] WWEA, "World wind energy report 2010," tech. rep., World Wind Energy Association, Bonn, Germany, 2011.

[2] P. Gipe, "Noise from small wind turbines: An unaddressed issue." www.wind-works.org/articles/noiseswt.html, viewed (2/1/2012), 2003.

[3] Encraft, "Microair - a catalyst for change in UK energy culture?." http://www.warwickwindtrials.org.uk/index.html, viewed (28/12/2011), 2007.

[4] Encraft, "Warwick microwind trial project - final report," tech. rep., Encraft, http://www.warwickwindtrials.org.uk/2.html, viewed (28/12/2011), 2009.

[5] A. Collar, "The expanding domain of aeroelasticity," Journal of the Royal Aeronautical Society, vol. L, pp. 613 - 636, August 1946.

[6] R. Bisplinghoff, H. Ashley, and R. Halfman, Aeroelasticity. Mineola, New York: Dover Publications, 1955.

[7] J. J. Allen and A. J. Smits, "Energy harvesting eel," Journal of Fluids and Structures, vol. 15, no. 3-4, pp. 629-640, 2001.

[8] E. Naudascher and D. Rockwell, "Oscillator-model approach to the identification and assessment of flow-induced vibrations in the system," Journal of Hydraulic Research, vol. 18, pp. 59-82, 1980.

[9] E. Naudascher and D. Rockwell, Flow-Induced Vibrations: An Engineering Guide. Rotterdam: A. A. Balkema, 1994.

[10] T. Theodorsen, "General theory of aerodynamic instability and the mechanism of flutter," Tech. Rep. No. 496, National Advisory Committee for Aeronautics, 1935.

[11] A. Kornecki, E. H. Dowell, and J. O'Brien, "On the aerodynamic instability of two-dimensional panels in uniform incompressible flow," Journal of Sound and Vibration, vol. 47, no. 2, pp. 163-178, 1976.

[12] L. Huang, "Flutter of cantilevered plates in axial flow," Journal of Fluids and Structures, vol. 9, pp. 127-147, 1995.

[13] M. Argentina and L. Mahadevan, "Fluid-flow-induced flutter of a flag," Proceedings of the National Academy of Sciences of the United States of America, vol. 102, no. 6, pp. 1829-34, 2005.

[14] A. Deivasigamani, J. McCarthy, S. Watkins, S. John, and F. Coman, "Flow-induced flutter of slender cantilever high-compliance plates," in 28th International Congress Of The Aeronautical Sciences (Paper No. 863), ICAS 2012, September 23 - September 28, (Brisbane, Australia), 2012.

[15] Z. Jun, S. Childress, A. Libchaber, and M. Shelley, "Flexible filaments in a flowing soap film as a model for one-dimensional flags in a two-dimensional wind," Nature, vol. 408, pp. 835-9, 2000.

[16] M. Hariri, S. John, and P. Trivailo, "Modelling piezoelectric actuation during structural flutter," in ASME Conference on Smart Materials, Adaptive Structures and Intelligent Systems, SMASIS2009, September 21 - September 23, vol. 1, (Oxnard, CA, United states), pp. 33-43, American Society of Mechanical Engineers, 2009.

[17] N. Yamaguchi, K. Yokota, and Y. Tsujimoto, "Flutter limits and behaviors of a flexible thin sheet in high-speed flow-I: analytical method for prediction of the sheet behavior," ASME Journal of Fluids Engineering, vol. 122, pp. 65-73, 2000.

[18] B. S. H. Connell and D. K. P. Yue, "Flapping dynamics of a flag in a uniform stream," Journal of Fluid Mechanics, vol. 581, pp. 33-67, 2007.

[19] S. Alben and M. J. Shelley, "Flapping states of a flag in an inviscid fluid: Bistability and the transition to chaos," Physical Review Letters, vol. 100, no. 7, pp. 074301-1 - 4, 2008

[20] P. Bearman, "Vortex shedding from oscillating bluff-bodies," Annual Review of Fluid Mechanics, vol. 16, pp. 195-222, 1984.

[21] R. Blevins, Flow-Induced Vibrations. New York: Van Nostrand Reinhold, 2 ed., 1990.

[22] A. Roshko, "On the development of turbulent wakes from vortex streets," tech. rep., National Advisory Committee for Aeronautics, Washington DC, USA, 1954a.

[23] A. Roshko, "On the drag and shedding frequency of two-dimensional bluff bodies," Tech. Rep. 3168, NACA, 1954b. 
[24] S. E. Ramberg, "The effects of yaw and finite length upon the vortex wakes of stationary and vibrating circular cylinders," Journal of Fluid Mechanics, vol. 128, pp. 81-107, March 1983.

[25] M. Zhao, L. Cheng, and T. Zhou, "Direct numerical simulation of three-dimensional flow past a yawed circular cylinder of infinite length," Journal of Fluids and Structures, vol. 25, no. 5, pp. $831-847,2009$.

[26] C.W. Van Atta, "Experiments on vortex shedding from yawed circular cylinders," AIAA Journal, vol. 6, pp. 931-933, May 1968.

[27] A. Bibo, Investigation of Concurrent Energy Harvesting from Ambient Vibrations and Wind. PhD thesis, Clemson University, Department of Mechanical Engineering, South Carolina, USA, August 2014.

[28] W. Cady, Piezoelectricity: an introduction to the theory and applications of electromechanical phenomena in crystals. New York: Dover Publications, 1964

[29] Midé, "Midé.” http://www.mide.com/index.php, 2015

[30] R. N. Jazar and L. Dai, eds., Nonlinear Approaches in Engineering Applications 2, ch. 6, pp. 169-211. New York: Springer Science+Business Media, 2014

[31] D. L. Churchill, M. J. Hamel, C. P. Townsend, and S. W. Arms, "Strain energy harvesting for wireless sensor networks," in Smart Structures and Materials 2003. Smart Electronics, MEMS, BioMEMS, and Nanotechnology, 3-5 March, vol. 5055, (USA), pp. 319-27, SPIE-Int. Soc. Opt. Eng, 2003.

[32] Q. Zhang, V. Bharti, G. Kavarnos, and M. Schwartz, "Poly-vinylidene fluoride (PVDF) and its copolymers," in Encyclopedia of smart materials, vol. 1-2, pp. 807-825, Wiley, 2002.

[33] Smart Material, "Macro fiber composite.” www.smart-material.com/MFC-product-main.html, 2015.

[34] Measurement Specialties, Inc., "Piezo film product guide and price list." http://www.meas-spec.com/piezo-film-sensors.aspx, 2015.

[35] L. Tang, M. P. Païdoussis, and J. Jiang, "Cantilevered flexible plates in axial flow: Energy transfer and the concept of flutter-mill," Journal of Sound and Vibration, vol. 326, no. 1-2, pp. 263 - 276, 2009.

[36] J. Dunnmon, S. Stanton, B. Mann, and E. Dowell, "Power extraction from aeroelastic limit cycle oscillations," Journal of Fluids and Structures, vol. 27, no. 8, pp. $1182-1198,2011$.

[37] D. T. Akcabay and Y. L. Young, "Hydroelastic response and energy harvesting potential of flexible piezoelectric beams in viscous flow," Physics of Fluids, vol. 24, no. 5, pp. 054106-1-19, 2012

[38] W. P. Robbins, D. Morris, I. Marusic, and T. O. Novak, "Wind-generated electrical energy using flexible piezoelectric materials," in Proceedings of IMECE 2006, vol. 14050, pp. 581-590, American Society of Mechanical Engineers, 2006.

[39] R. Dickson, New Concepts in Renewable Energy. Lulu Enterprises, Inc., 2008.

[40] S. Li and H. Lipson, "Vertical-stalk flapping-leaf generator for wind energy harvesting," in ASME Conference on Smart Materials, Adaptive Structures and Intelligent Systems, SMASIS2009, September 21 - September 23, vol. 2, (Oxnard, CA, United states), pp. 611-619, American Society of Mechanical Engineers, 2009.

[41] S. Li, J. Yuan, and H. Lipson, “Ambient wind energy harvesting using cross flow fluttering," Journal of Applied Physics, vol. 109, no. 026104, 2011.

[42] A. Deivasigamani, J. McCarthy, S. John, S. Watkins, F. Coman, and P. Trivailo, "Flutter of cantilevered interconnected beams with variable hinge positions," Journal of Fluids and Structures, vol. 38, pp. 223-237, April 2012.

[43] M. Bryant, E. Garcia, and E. Wolff, "Aeroelastic flutter energy harvester design: the sensitivity of the driving instability to system parameters," Smart Materials and Structures, vol. 20, no. 12, pp. 1-12, 2011.

[44] J. McCarthy, A. Deivasigamani, S. John, S. Watkins, and F. Coman, "The effect of the configuration of the amplification device on the power output of a piezoelectric strip," in ASME Conference on Smart Materials, Adaptive Structures and Intelligent Systems (Paper No. 7951), SMASIS2012, September 19 - September 21, (Stone Mountain, Georgia, USA), American Society of Mechanical Engineers, 2012.

[45] J. McCarthy, Energy Capture From Ambient Flows Using Piezoelectric Flutter Harvesters. PhD thesis, RMIT University School of Aerospace, Mechanical and Manufacturing Engineering, Melbourne, Australia, January 2015.

[46] M. Bryant, R. Mahtani, and E. Garcia, "Synergistic wake interactions in aeroelastic flutter vibration energy harvester arrays," in ASME Conference on Smart Materials, Adaptive Structures and Intelligent Systems, SMASIS2011, September 18 - September 21, (Scottsdale, AZ, United States), American Society of Mechanical Engineers, 2011.

[47] M. Bryant, R. L. Mahtani, and E. Garcia, "Wake synergies enhance performance in aeroelastic vibration energy harvesting," Journal of Intelligent Material Systems and Structures, vol. 23, no. 10, pp. $1131-1141,2012$.

[48] J. McCarthy, A. Deivasigamani, S. Watkins, S. John, and F. Coman, "A parametric study of wind-induced flutter of piezoelectric patches for energy harvesting," in 15th Australian International Aerospace Congress (Paper No. 194), AIAC15, (Melbourne, Australia), February 2013.

[49] J. McCarthy, A. Deivasigamani, S. Watkins, S. John, F. Coman, and P. Petersen, "Downstream flow structures of a fluttering piezoelectric energy harvester," Experimental Thermal and Fluid Science, vol. 51, pp. 279-290, 2013.

[50] J. McCarthy, A. Deivasigamani, S. Watkins, S. John, F. Coman, and P. Petersen, "On the visualisation of flow structures downstream of fluttering piezoelectric energy harvesters in a tandem configuration," Experimental Thermal and Fluid Science, vol. 57, pp. 407-419, 2014.

[51] A. Deivasigamani, J. McCarthy, S. John, S. Watkins, and F. Coman, "Energy harvesting from fluid flow using coupled bending-torsion flutter of a thin composite piezoelectric beam," in 19th International Conference on Composite Materials, (Montreal, Canada), July 2013.

[52] A. Deivasigamani, J. McCarthy, S. John, S. Watkins, F. Coman, and P. Trivailo, "Energy harvesting from fluid flow using coupled bendingtorsional vibrations," Journal of Modern Applied Sciences, vol. 8, pp. 106-126, 2014.

[53] A. Abdelkefil, F. Najar, A. Nayfeh, and A. Ben, "An energy harvester using piezoelectric cantilever beams undergoing coupled bendingtorsion vibrations," Journal of Smart Materials and Structures, vol. 20, no. 115007, 2011

[54] S. Pobering and N. Schwesinger, "A novel hydropower harvesting device," in International Conference on MEMS, NANO and Smart Systems, ICMENS 2004, August 25 - August 27, (Banff, Alta., Canada), pp. 480-485, IEEE Computer Society, 2004.

[55] H. Akaydan, N. Elvin, and Y. Andreopoulis, "Wake of a cylinder: a paradigm for energy harvesting with piezoelectric materials," Experiments in Fluids, vol. 49, pp. 291-304, 2010.

[56] H. D. Akaydan, N. Elvin, and Y. Andreopoulis, "Energy harvesting from highly unsteady fluid flows using piezoelectric materials," Journal of Intelligent Material Systems and Structures, vol. 21, pp. 1263-1278, 2010. 
[57] T. Tsutsui, T. Igarashi, and K. Kamemoto, "Interactive flow around two circular cylinders of different diameters at close proximity. experiment and numerical analysis by vortex method," Journal of Wind Engineering and Industrial Aerodynamics, vol. 69-71, pp. 279 - $291,1997$.

[58] S. Kang, "Uniform-shear flow over a circular cylinder at low Reynolds numbers," Journal of Fluids and Structures, vol. 22, no. 4, pp. 541-555, 2006.

[59] M. Zhao, L. Cheng, B. Teng, and G. Dong, "Hydrodynamic forces on dual cylinders of different diameters in steady currents," Journal of Fluids and Structures, vol. 23, no. 1, pp. 59-83, 2007.

[60] H. Y. Dong and N. P. Jones, "Characterization of flow oblique to a circular cylinder with low aspect ratio using 3-D detached eddy simulation," Journal of Wind Engineering and Industrial Aerodynamics, vol. 99, no. 11, pp. 1117 - 1125, 2011.

[61] O. Goushcha, N. Elvin, and Y. Andreopoulos, "Interactions of vortices with a flexible beam with applications in fluidic energy harvesting," Applied Physics Letters, vol. 104, no. 2, pp. 021919-1-4, 2014.

[62] A. Elzawawy, Time resolved particle image velocimetry techniques with continuous wave laser and their application to transient. PhD thesis, City University of New York, New York, 2012.

[63] W. B. Hobbs and D. L. Hu, "Tree-inspired piezoelectric energy harvesting," Journal of Fluids and Structures, vol. 28, pp. 103 - 114, 2012.

[64] T. Burton, D. Sharpe, N. Jenkins, and E. Bossanyi, Wind Energy Handbook. John Wiley \& Sons, 2001.

[65] J. Hobeck and D. J. Inman, "Energy harvesting from turbulence-induced vibration in airflow: Artificial piezoelectric grass concept," in 2011 ASME Conference on Smart Materials, Adaptive Structures and Intelligent Systems, SMASIS2011, September 18 - September 21, (Scottsdale, AZ, United States), American Society of Mechanical Engineers, 2011.

[66] J. D. Hooper and A. R. Musgrove, "Reynolds stress, mean velocity, and dynamic static pressure measurement by a four-hole pressure probe," Experimental Thermal and Fluid Science, vol. 15, pp. 375-383, 1997.

[67] D. Walshe, "Wind-excited oscillation of structures," Her Majesty's Stationary Office, 1972.

[68] D. Walshe, R. Thuillier, and U. Lappe, "Wind and temperature profile characteristics from observations on a $1400 \mathrm{ft}$ tower," Journal of Applied Meteorology, vol. 3, pp. 299-306, 1964.

[69] R. Nelson, Flight Stability and Automatic Control. New York: McGraw-Hill, 2 ed., 1998.

[70] J. Holmes, Wind Loading of Structures. London: Spon Press, 2001

[71] J. Owen, M. Sterling, D. Hargreaves, and C. Baker, eds., Fifty Years of Wind Engineering: Prestige Lectures from the Sixth European and African Conference on Wind Engineering, (United Kingdom), University of Cambridge, July 7-11 2012.

172] B. Etkin, "Turbulent wind and its effect on flight," AIAA Journal of Aircraft, vol. 18, no. 5, pp. 327-345, 1981.

[73] O. Sutton, Micrometeorology. New York: McGraw-Hill, 1953.

[74] I. Van Der Hoven, "Power spectrum of horizontal wind speed in the frequency range from 0.0007 to 900 cycles per hour," Journal of Meteorology, vol. 14, pp. 160-164, 1957.

[75] T. Lawson, Wind Effects on Buildings. London: Applied Science Publishers Ltd., 1980.

[76] W. Melbourne, Bluff Body Aerodynamics for Wind Engineering; A State of the Art in Wind Engineering. Wiley Eastern Ltd., 1994.

[77] ESDU, Characteristics of Atmospheric Turbulence Near the Ground. Part 1: Definitions and General Information. No. 74030, London: ESDU International, 1974.

[78] ESDU, Characteristics of Atmospheric Turbulence Near the Ground. Part 2: Single Point Data for Strong Winds (Neutral Atmosphere). No. 85020, London: ESDU International, 1985.

[79] ESDU, Characteristics of Atmospheric Turbulence Near the Ground. Part 3: Variations in Space and Time for Strong Winds (Neutral Atmosphere). No. 86010, London: ESDU International, 1986.

[80] Australian Bureau of Meteorology, “Climate Data Online.” www.bom.gov.au, 2015

[81] R. Flay, Structure of a Rural Atmospheric Boundary Layer near the Ground. PhD thesis, Department of Mechanical Engineering, University of Canterbury, New Zealand, November 1978

[82] J. McCarthy, S. Watkins, A. Deivasigamani, S. John, and F. Coman, "A study of piezoelectric energy harvester in smooth and turbulent flows: how might they perform in real-world conditions?," in 19th Australasian Fluid Mechanics Conference, (Melbourne, Australia), December 2014.

[83] J. McCarthy, S. Watkins, A. Deivasigamani, S. John, and F. Coman, "An investigation of fluttering piezoelectric energy harvesters in off-axis and turbulent flows," Journal of Wind Engineering and Industrial Aerodynamics, vol. 136, pp. 101 - 113, 2015.

[84] G. W. Taylor, J. R. Burns, S. M. Kammann, W. B. Powers, and T. R. Welsh, "The energy harvesting eel: A small subsurface ocean/river power generator," IEEE Journal of Oceanic Engineering, vol. 26, no. 4, pp. 539-547, 2001.

[85] J. L. Cao, S. John, and T. Molyneaux, "Energy transduction analysis of piezoelectric-based vibration control of smart structures," ANZIAM Journal, vol. 47, pp. C733-C759, 2007.

[86] S. Roundy and Y. Zhang, "Toward self-tuning adaptive vibration based micro-generators," in Proceedings of SPIE, Smart Structures, Devices, and Systems II (S. F. Al-Sarawi, ed.), vol. 5649, (Sydney, Australia), pp. 373-384, 2005

[87] S. R. Anton and H. A. Sodano, "A review of power harvesting using piezoelectric materials (2003-2006)," Smart Materials and Structures, vol. 16, pp. R1-R21, 2007.

[88] A. Deivasigamani, Piezoelectric energy harvesting from wind-induced flutter. PhD thesis, RMIT University School of Aerospace, Mechanical and Manufacturing Engineering, Melbourne, Australia, January 2015.

[89] A. Betz, Introduction to the Theory of Flow Machines. Oxford: Pergamon Press, 1966.

[90] M. Bryant, A. Schlichting, and E. Garcia, "Toward efficient aeroelastic energy harvesting: Device performance comparisons and improvements through synchronized switching," in Proceedings of SPIE, Active and Passive Smart Structures and Integrated Systems (H. Sodano, ed.), vol. 8688, pp. 868807-1-10, 2013.

[91] J. Sirohi and R. Mahadik, "Piezoelectric wind energy harvester for low-power sensors," Journal of Intelligent Material Systems and Structures, vol. 22, no. 18, pp. 2215-2228, 2011.

[92] R. Myers, M. Vickers, H. Kim, and S. Priya, "Small scale windmill," Applied Physics Letters, vol. 90, pp. 054106-1 - 3, 2007.

[93] M. Raju, "Energy Harvesting: ULP Meets Energy Harvesting." Texas Instruments White Paper, 2008.

[94] Professional Plastics, "PVDF Film Pricing.” http://www.professionalplastics.com/PVDFFILM, viewed (30/04/2015), 2015. 
[95] M. van der Hoeven, "Technology roadmap: Solar photovoltaic energy,” tech. rep., International Energy Agency, 2014.

96] A. Deivasigamani, J. McCarthy, S. John, S. Watkins, and F. Coman, "Proximity effects of piezoelectric energy harvesters in fluid flow," in 29th Congress of the International Council of the Aeronautical Sciences, ICAS 2014, September 7 - 12, (St. Petersburg, Russia), 2014. 